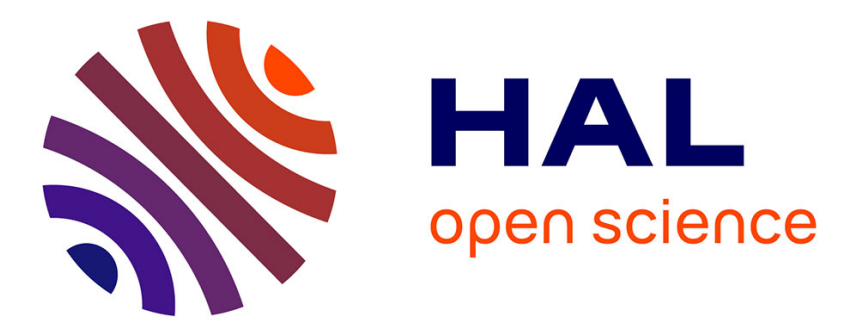

\title{
Shear-flow trapped-ion-mode interaction revisited. I. Influence of low-frequency zonal flow on ion-temperature-gradient driven turbulence
}

\author{
Alain Ghizzo, F. Palermo
}

\section{- To cite this version:}

Alain Ghizzo, F. Palermo. Shear-flow trapped-ion-mode interaction revisited. I. Influence of lowfrequency zonal flow on ion-temperature-gradient driven turbulence. Physics of Plasmas, 2015, 22 (8), pp.82303 - 82304. 10.1063/1.4928102 . hal-01285771

HAL Id: hal-01285771

https://hal.science/hal-01285771

Submitted on 20 Apr 2018

HAL is a multi-disciplinary open access archive for the deposit and dissemination of scientific research documents, whether they are published or not. The documents may come from teaching and research institutions in France or abroad, or from public or private research centers.
L'archive ouverte pluridisciplinaire HAL, est destinée au dépôt et à la diffusion de documents scientifiques de niveau recherche, publiés ou non, émanant des établissements d'enseignement et de recherche français ou étrangers, des laboratoires publics ou privés. 


\section{Shear-flow trapped-ion-mode interaction revisited. I. Influence of low-frequency zonal flow on ion-temperature-gradient driven turbulence}

A. Ghizzo, and F. Palermo

Citation: Physics of Plasmas 22, 082303 (2015); doi: 10.1063/1.4928102

View online: https://doi.org/10.1063/1.4928102

View Table of Contents: http://aip.scitation.org/toc/php/22/8

Published by the American Institute of Physics

\section{Articles you may be interested in}

Shear-flow trapped-ion-mode interaction revisited. II. Intermittent transport associated with low-frequency zonal flow dynamics

Physics of Plasmas 22, 082304 (2015); 10.1063/1.4928103

Shear flow instabilities induced by trapped ion modes in collisionless temperature gradient turbulence

Physics of Plasmas 22, 042304 (2015); 10.1063/1.4916770

Self-generated zonal flows in the plasma turbulence driven by trapped-ion and trapped-electron instabilities

Physics of Plasmas 22, 102309 (2015); 10.1063/1.4933358

Global gyrokinetic simulations of trapped-electron mode and trapped-ion mode microturbulence

Physics of Plasmas 22, 082302 (2015); 10.1063/1.4927920

A simple model for electron dissipation in trapped ion turbulence

Physics of Plasmas 24, 012511 (2017); 10.1063/1.4974269

Validation of nonlinear gyrokinetic simulations of L- and I-mode plasmas on Alcator C-Mod

Physics of Plasmas 24, 056104 (2017); 10.1063/1.4977466

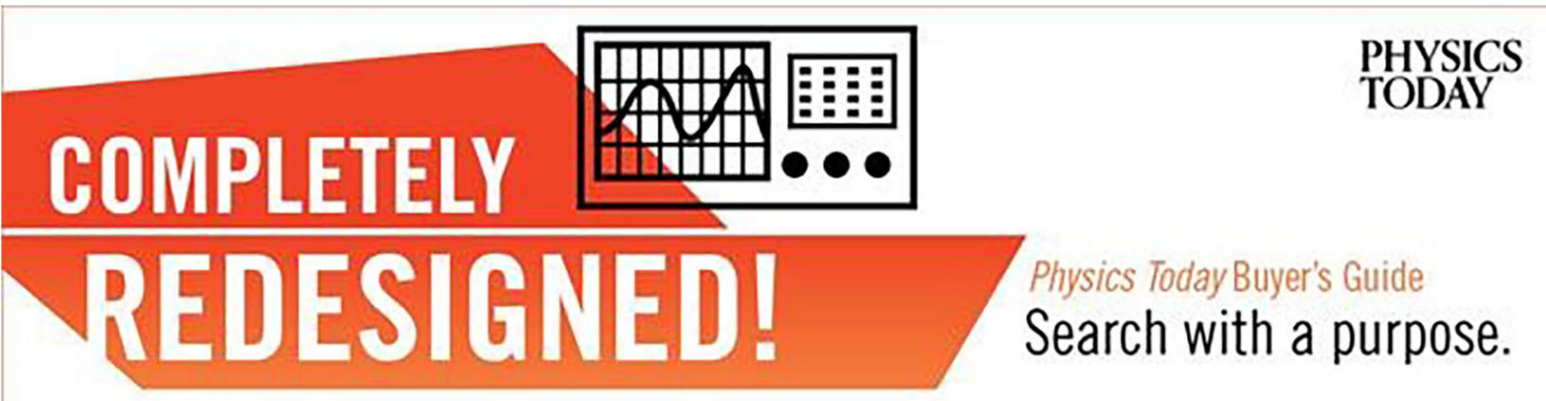




\title{
Shear-flow trapped-ion-mode interaction revisited. I. Influence of low-frequency zonal flow on ion-temperature-gradient driven turbulence
}

\author{
A. Ghizzo ${ }^{1, a)}$ and F. Palermo ${ }^{2}$ \\ ${ }^{1}$ Institut Jean Lamour-UMR 7168, University of Lorraine, BP 239, F-54506 Vandoeuvre les Nancy, Lorraine, \\ France \\ ${ }^{2}$ LFMA—Ecole Centrale de Lyon, Université de Lyon, 60134 Ecully, France
}

(Received 3 April 2015; accepted 17 July 2015; published online 6 August 2015)

Collisionless trapped ion modes (CTIMs) turbulence exhibits a rich variety of zonal flow physics. The coupling of CTIMs with shear flow driven by the Kelvin-Helmholtz (KH) instability has been investigated. The work explores the parametric excitation of zonal flow modified by wave-particle interactions leading to a new type of resonant low-frequency zonal flow. The KH-CTIM interaction on zonal flow growth and its feedback on turbulence is investigated using semi-Lagrangian gyrokinetic Vlasov simulations based on a Hamiltonian reduction technique, where both fast scales (cyclotron plus bounce motions) are gyro-averaged. (C 2015 AIP Publishing LLC.

[http://dx.doi.org/10.1063/1.4928102]

\section{INTRODUCTION}

Turbulence due to drift waves is a ubiquitous feature of magnetically confined plasmas, such as those in tokamaks. Drift waves can be driven to be unstable, for example, by radial gradients both in density and temperature. In the studies of magnetized plasmas, theories and simulations have predicted that drift-wave turbulence can generate the mesoscale structures, such as zonal flows (ZFs), sheared flows, trapped ion modes (TIMs), but also streamers. These mesoscale structures, ZFs and streamers, have different behaviours and their impacts on turbulence-driven transport show strong contrasts. Motivated by the experimental discovery of a lowto-high (LH) transition ${ }^{1}$ in the plasma confinement characteristics, experiment and theory in the last decade have focused on whether the turbulence associated with the H-states might be regulated by interactions with ZFs. Central to all these enhanced confinement regimes, be they in the edge region or the core region of the tokamak plasma, is the generation of sheared flows or zonal flows, which are believed to be responsible for suppressing fluctuations and stabilizing the turbulence.

A clear indication of the key role played by ZFs was the recent observations in Ref. 2 at the Experimental Advanced Superconducting Tokamak (EAST) tokamak or in ASDEX Upgrade tokamak in Ref. 3, associated with a low frequency signal at a few kilohertz corresponding to coherent ZF oscillations preceding and following the $\mathrm{LH}$ transition. The signal was observed at a much lower frequency than the geodesic acoustic mode (GAM) with strong correlation of such an oscillation with the turbulent Reynolds stress. No coherent oscillation in the GAM frequency range was detected by the probes in these experiments in the H-mode regime. This brings us to an important question of the physical origin of these low-frequency oscillations while the ZF frequency is usually attributed to be zero in the hydrodynamical

\footnotetext{
a)alain.ghizzo@univ-lorraine.fr
}

approach. These experimental measurements indicate also that particles towards the lower divertor are modulated at the same low frequency value, e.g., $\leq 4 \mathrm{kHz}$ indicating strong correlations with trapped ions since standard GAMs are not excited (we can then postulate that circulating ions do not play a major role).

In contrast, streamers contribute to enhance the transport owing to their radial elongated structures. Streamers, observed in numerical simulation studies, appear to be closely associated with avalanche type transport events. Thus, theoretical understanding of such ZFs and streamers has first come from studies on the drift wave model but also in the gyrokinetic turbulence, e.g., the ion temperature gradient (ITG) mode, the electron temperature gradient (ETG) mode, and trapped electron mode (TEM).

ZFs are $E \times B$ flows with toroidally $(n=0)$ and poloidally $(m=0)$ symmetry but radially varying electric potential fluctuations with quasi zero frequency. In the toroidal geometry, Rosenbluth and Hinton ${ }^{4}$ have shown that the $n=0$ poloidal flows driven by the ITG turbulence, although modified by plasma polarization, are not linearly (Landau) damped by collisionless processes. Residual ZFs have been experimentally observed in the core of plasmas DIII-D tokamak in Ref. 5, while streamers were observed in Refs. 6 and 7. In Ref. 6 , the experiment was carried out in JIPP-T-IIU tokamak plasma with a nearly circular cross section and streamers were found in the bad curvature region, presenting ballooning-type characteristics.

ZFs have the ability to limit the radial size of turbulent vortices through the shear de-correlation mechanism ${ }^{8}$ and hence to regulate turbulent transport. The generation of ZFs in drift wave turbulence was first predicted by Hasegawa and Mima (HM) in Ref. 9 and by Charney ${ }^{10}$ in the case of the geostrophic vortex equation. Related models based on the HM model are the modified Hasegawa-Mima model (MHM) in Ref. 11, Hasegawa-Wakatani (HW) model for the study of plasma edge turbulence in Ref. 12, and modified HasegawaWakatani (MHW) model in Refs. 13-15. 
While considerable progress has been achieved in the understanding of the zonal flow physics (see for instance Refs. 16 and 17), many aspects of ZF dynamics remain nevertheless poorly understood. The generation of ZFs and their feedback to turbulence and transport are essentially nonlinear processes. ZFs are generated through the Reynolds stress in the drift wave turbulence but give no perpendicular heat nor particle transport. Nevertheless, since sheared flows can suppress the micro-turbulence, they play quite an important role in regulating turbulent transport. Thus, the selfgenerated zonal flow in plasma turbulence is now recognized as a key constituent of a drift-wave -ZF system.

Moreover, ZFs are also met in nature, and the most famous example is the strong longitudinal winds in the Jovian atmosphere. The physics of ZF formation was also studied within the geophysical fluid dynamics community, and Charney and Drazin ${ }^{18}$ have elucidated the nonlinear origin and dynamics of ZF using a momentum conservation theorem. Such a theorem was extended to plasma physics by Diamond et al. ${ }^{19}$

For the saturation of ZF growth in plasma turbulence, several mechanisms have been proposed. The KelvinHelmholtz (KH) instability is one of the candidates for a saturation mechanism of ZF in ITG turbulence (see Refs. 20 and 21). The complexity of the problem is linked, on the one hand by the linear and nonlinear aspects of $\mathrm{KH}$ modes and on the other hand by the nonlinear interactions among driftwave, ZFs, and KH modes. While substantial effort has been devoted to the study of zonal flow generation by drift wave or ITG modes, very little work has been published on zonal flows driven by pure TIMs or by their counterpart of TEMs when electron dynamics is taken into account.

TIMs are a prototype of kinetic mode since they are driven through the resonant interaction between a wave and trapped ions through their precession motion. Although TIMs have been studied by Kadomtsev and Pogutse ${ }^{22} 40$ years ago (usually referred as dissipative modes due to collisions), collisionless TIMs (CTIMs) are known to be subject to interchange-type turbulence. While dissipative modes propagate in the electron direction, collisionless modes possess two distinct branches. The first one is thus analogous to interchange-like mode (and therefore to ITG modes). But TIMs can also be driven by resonance since they propagate in the ion diamagnetic direction. Thus, the resonance with precessing trapped ions becomes important. Their hybridkinetic character reveals new features, in particular, the property to generate (nonlinear) streamers (see Refs. 23 and 24) by resonant interactions and to couple with $\mathrm{ZF}$ and $\mathrm{KH}$ modes. In revising the theory, we will address how $\mathrm{ZF}$ is modified in the nonlinear (kinetic) resonant regime by its coupling with TIMs leading to a low-frequency oscillatory behaviour in time, allowing enhanced resonant wave-particle interactions of kinetic nature, a process that is different from the standard Reynolds stress met in hydrodynamics. It is in light of these considerations that we undertake here an investigation of the nonlinear dynamics of the trapped ion mode in the presence of $\mathrm{KH}$ instability.

The remainder of this paper is organized as follows. In Sec. II, we present the nonlinear equations of the trapped-ion model and give in Sec. III the main properties of the model in terms of energy conservation and we build the equations of first moments of the reduced Vlasov equation. In particular, we derive and analyze the equations of the mean quantities $\langle\phi\rangle_{\alpha}$ and $\langle P\rangle_{\alpha}$ describing the zonal flow in the presence of interchange turbulence. In Sec. IV, we solve the Vlasov equations numerically using a semi-Lagrangian scheme and present the results for the interchange turbulence, while the $\mathrm{KH}$ regime is studied in Sec. V. Section VI contains the summary.

\section{NONLINEAR MODEL EQUATIONS}

TIMs were obtained by averaging over the fast scales, cyclotron plus bounce motions. This task is made easier in the framework of the Hamiltonian formalism using actionangle variables. The population of trapped ions is then described by a distribution function $\bar{f}=\bar{f}_{E, K}(\psi, \alpha, t)$, where $E$ and $\kappa$ are considered as adiabatic invariants. The lowfrequency response for TIM is obtained by making a phaseangle average over the cyclotron phase and the bounce motion (the "banana" orbit) leading to invariance of the total energy $E=\frac{1}{2} m v_{G \|}^{2}+\mu B\left(\boldsymbol{x}_{G}\right)$ and of the so-called adiabatic invariant $\mu=\frac{m v_{G}^{2}}{2 B\left(x_{G}\right)}$. Here, the label $G$ is a conventional notation that refers to the guiding centre and $\boldsymbol{x}_{G}$ refers to $(r, \theta)$ polar coordinates. By introducing the pitch angle parameter $\kappa$ defined by $\kappa^{2}=\sin ^{2}\left(\frac{\theta_{0}}{2}\right)=\frac{1-\lambda}{2 \varepsilon \lambda}$, where $\lambda=\frac{\mu B_{0}}{E}$ and $\varepsilon=\frac{r}{R_{0}}$ is the inverse aspect ratio, trapped particles are characterized by $\kappa<1$, while $\kappa>1$ corresponds to passing ions. Following the work of Kadomtsev and Pogutse, ${ }^{22}$ the bounce and precession frequencies are given, respectively, by the following relations:

$$
\begin{gathered}
\omega_{b}=\sqrt{\frac{2 E}{m}} \frac{1}{q_{0} R_{0}} \bar{\omega}_{b}(\kappa) \quad \text { with } \quad \bar{\omega}_{b}(\kappa) \simeq \frac{\pi \sqrt{\varepsilon}}{2 \sqrt{2} K(\kappa)}, \\
\omega_{d} E=\frac{q_{0} E}{e r_{0} R_{0} B_{0}} \bar{\omega}_{d}(\kappa ; s) \quad \text { with } \\
\bar{\omega}_{d}(\kappa ; s)=\frac{2 E(\kappa)}{K(\kappa)}-1+4 s\left(\frac{E(\kappa)}{K(\kappa)}+\kappa^{2}-1\right),
\end{gathered}
$$

where $s=\frac{r_{0}}{q_{0}}\left(\frac{d q}{d r}\right)_{r_{0}}$ is the magnetic shear and $K(\kappa)$ and $E(\kappa)$ are the complete elliptic integral of the first and second kind, respectively. Finally, the two important variables are the precession angle $\alpha=\varphi-q_{0} \theta$ and the poloidal flux $\psi$ related to the third "action quantity" $J_{3}=M=e \psi+m R\left(\boldsymbol{x}_{G}\right) v_{G 0}$ $\equiv e \psi\left(r_{0}\right)$, assumed to be not an adiabatic invariant. The resulting gyrokinetic Vlasov equation is then given by

$$
\frac{\partial \bar{f}}{\partial t}+\omega_{d}(\kappa) E \frac{\partial \bar{f}}{\partial \alpha}+[\bar{\phi}, \bar{f}]=\partial_{\psi}\left(D(\psi) \partial_{\psi} \bar{f}\right),
$$

where $[.,$.$] is the usual Poisson bracket defined by [g, f]=\partial_{\psi}$ $g \partial_{\alpha} f-\partial_{\alpha} g \partial_{\psi} f$ and $\bar{\phi}=J_{0} \phi$ is the bounce average electric potential. Assuming an adiabatic response for electrons, the electron-neutrality condition $\delta n_{e}=\delta n_{i}$ reads as 


$$
C_{e}\left(\phi-\langle\phi\rangle_{\alpha}\right)-C_{i} \bar{\nabla}^{2} \phi=\overline{\bar{n}}_{i}-n_{0},
$$

where

$$
\bar{\nabla}^{2}=\rho_{s}^{2} \partial_{\alpha}^{2}+\delta_{b}^{2} \partial_{\psi}^{2}
$$

with the asymmetry between the $\psi$ and $\alpha$ directions, which close the system of Eqs. (3) and (4) in a self-consistent way. Here, $n_{0}$ denotes the passing ion density. The gyro-average operator $J_{0}$ was approximated by the Pade's relation giving to

$$
J_{0}=\left(1-\frac{E}{4} \delta_{b}^{2} \partial_{\psi}^{2}\right)^{-1}\left(1-\frac{E}{4} \rho_{s}^{2} \partial_{\alpha}^{2}\right)^{-1} .
$$

The gyro-averaged pseudo-density of trapped ions used in Eq. (3) is given by Eq. (A2) of the Appendix. Here, $C_{e}$ and $C_{i}$ are constants accounting for the fraction of trapped particles $f_{p}$ and for the ratio $\tau$ of ion to electron temperatures $C_{e}=(1+\tau) / f_{p}$ and $C_{i}=C_{e} f_{p} / \tau, \tau=T_{i} / T_{e}$. Trapped ion turbulence develops on length scale of the order of the banana width $\delta_{b}$ and time scale determined by $\omega_{d 0}^{-1}$, where $\omega_{d 0}=\frac{q_{0} T_{0}}{e r_{0} R_{0} B_{0}}$. Here, $\delta_{b}$ is constant (we have neglected the dependence in $\kappa$ ) and is found close to $\frac{\rho_{s} q_{0}}{\sqrt{\varepsilon}}$, where $\rho_{s}$ is the Larmor radius. $B_{0}$ is the minimal value of the magnetic field amplitude $B$ at $\theta=0$ ( $R=R_{0}$ being then the major radius). For more details concerning the TIM model, see also Refs. 23,25 , and 26 .

\section{PROPERTIES OF THE TRAPPED ION MODEL}

\section{A. Energy conservation law}

In the following, we consider the general properties of the energy conservation of the trapped ion model in order to elucidate the processes of energy transfer to zonal flow. First, since (3) is a Vlasov kinetic equation, $f$ is conserved (provided of course that dissipation is zero). We assume that the gyroaverage operator $J_{0}$ is equal to unity. In that case, the smoothed distribution function $\bar{f}=\bar{f}_{\kappa, E}(\psi, \alpha, t)=J_{0} f$ becomes the distribution $f$ and we consider conservation of particles and energy. By integrating the Vlasov equation (3) over the parameters $\kappa$ and $E$, we obtain the following relation:

$$
\frac{\partial n}{\partial t}+[\phi, n]+\frac{3}{2} \omega_{d 0} \frac{\partial P^{(1)}}{\partial \alpha}=\frac{\partial}{\partial \psi}\left(D(\psi) \frac{\partial n}{\partial \psi}\right)
$$

where $n$ and $P^{(1)}$ are the ion density and the ion pressure, respectively, determined by taking into account the contribution of the normalized drift frequency $\bar{\omega}_{d}(\kappa ; s)$ for the trapped ion population noted by $\kappa$. We have thus,

$$
\begin{aligned}
n(\psi, \alpha, t)=\int_{0}^{1} d \kappa \kappa & K(\kappa) \frac{2}{\sqrt{\pi}} \int_{0}^{+\infty} d E \sqrt{E} f_{\kappa, E}(\psi, \alpha, t), \\
\frac{3}{2} P^{(n)}(\psi, \alpha, t)= & \int_{0}^{1} d \kappa \kappa K(\kappa) \bar{\omega}_{d}^{n}(\kappa ; s) \\
& \times \frac{2}{\sqrt{\pi}} \int_{0}^{+\infty} d E \sqrt{E} E f_{\kappa, E}(\psi, \alpha, t) .
\end{aligned}
$$

By denoting $\frac{d n}{d t}=\frac{\partial n}{\partial t}+[\phi, n]$, Eq. (7) reads as

$$
\frac{d n}{d t}+\frac{3}{2} \omega_{d 0} \frac{\partial P^{(1)}}{\partial \alpha}=\frac{\partial}{\partial \psi}\left(D(\psi) \frac{\partial n}{\partial \psi}\right) .
$$

Thus, the density of trapped ions is conserved since

$$
\iint \frac{d \alpha}{2 \pi} d \psi \frac{\partial n}{\partial t}=0
$$

To show the energy conservation, we multiply Eq. (3) (when $D(\psi) \rightarrow 0$ ) by the quantity $\omega_{d}(\kappa) E \psi$ (with $\omega_{d}=\omega_{d 0} \bar{\omega}_{d}$ ) and integrate over $\kappa$ and $E$. Note that in the Hamiltonian formulation of the Vlasov equation given in (3), the corresponding Hamiltonian is $H=\omega_{d}(\kappa) E \psi+J_{0} \phi$. For the second order moment of (3), we have

$$
\begin{aligned}
\frac{d \Theta}{d t} & +\partial_{\alpha}\left(\omega_{d 0}^{2} M_{2}^{(2)}+\frac{3}{2} \omega_{d 0} \phi P^{(1)}\right)-\phi \partial_{\alpha}\left(\frac{3}{2} \omega_{d 0} P^{(1)}\right) \\
& =\frac{3}{2} \omega_{d 0} \psi \partial_{\psi}\left(D \frac{\partial P^{(1)}}{\partial \psi}\right) .
\end{aligned}
$$

In Eq. (12), we have introduced the kinetic energy density $\Theta$ and the second order $M_{2}^{(n)}$ moment defined, respectively, by

$$
\begin{aligned}
& \Theta=\psi \omega_{d 0} \int_{0}^{1} d \kappa \kappa K(\kappa) \bar{\omega}_{d}(k ; s) \frac{2}{\sqrt{\pi}} \int_{0}^{+\infty} d E \sqrt{E} E f_{\kappa, E}, \\
& M_{2}^{(n)}=\psi \omega_{d 0} \int_{0}^{1} d \kappa \kappa K(\kappa) \bar{\omega}_{d}^{n}(k ; s) \frac{2}{\sqrt{\pi}} \int_{0}^{+\infty} d E \sqrt{E} E^{2} f_{\kappa, E} .
\end{aligned}
$$

Substituting (10) and (4) in Eq. (12) and considering the potential into the form $\phi=\langle\phi\rangle_{\alpha}+\delta \phi$ (with $\langle\delta \phi\rangle_{\alpha}=0$ ), we obtain, after integrating over the variables $\alpha$ and $\psi$

$$
\begin{aligned}
& \frac{d E_{c}}{d t}+\frac{d E_{Z F}}{d t}+\frac{d E_{\text {turb }}}{d t}=\text { losses } \\
= & \iint \frac{d \alpha}{2 \pi} d \psi\left(\frac{3}{2} \omega_{d 0} \psi \frac{\partial}{\partial \psi}\left(D(\psi) \frac{\partial P^{(1)}}{\partial \psi}\right)\right. \\
+ & \left.\phi \frac{\partial}{\partial \psi}\left(D(\psi) \frac{\partial n}{\partial \psi}\right)\right) .
\end{aligned}
$$

Thus, the total energy can be decomposed into three distinct parts: the kinetic energy $E_{c}$, the zonal flow contribution denoted by $E_{Z F}$, and the turbulence contribution $E_{\text {turb. Both }}$ quantities are defined, respectively, by

$$
\begin{gathered}
E_{c}=\iint \frac{d \alpha}{2 \pi} d \psi \Theta \\
E_{Z F}=\frac{1}{2} C_{i} \delta_{b}^{2} \iint \frac{d \alpha}{2 \pi} d \psi\left(\frac{\partial\langle\phi\rangle_{\alpha}}{\partial \psi}\right)^{2}, \\
E_{\text {turb }}=\iint \frac{d \alpha}{2 \pi} d \psi\left(\frac{1}{2} C_{e} \delta \phi^{2}+\frac{1}{2} C_{i}|\bar{\nabla} \delta \phi|^{2}\right) .
\end{gathered}
$$




\section{B. Gyro-average operator driven effects on energy conservation}

The difficulty can now to be seen by noting that $M_{2}^{(n)}$ in Eq. (14) or the pressure $P^{(n)}$ in Eq. (10) depend on the quantity $\bar{\omega}_{d}^{n}(\kappa)$. Thus, to consider energy conservation, we need to compute both of these terms and to take into account the corrections introduced by the gyro-average operator $J_{0}$ approximated here by $J_{0} \simeq 1+\frac{E}{4} \bar{\triangle}$. The different successive moments of the reduced Vlasov equation (3) become

$$
\begin{gathered}
\frac{\partial \bar{n}}{\partial t}+[\phi, \bar{n}]+\frac{3}{2} \omega_{d 0} \partial_{\alpha}\left(\bar{P}^{(1)}\right)+\frac{3}{8}\left[\bar{\triangle} \phi, \bar{P}^{(0)}\right] \\
=\partial_{\psi}\left(D(\psi) \partial_{\psi} \bar{n}\right) \\
\frac{3}{2}\left(\frac{\partial \bar{P}^{(0)}}{\partial t}+\left[\phi, \bar{P}^{(0)}\right]\right)+\omega_{d 0} \partial_{\alpha}\left(\bar{M}_{2}^{(1)}\right) \\
+\left[\bar{\triangle} \phi, \frac{1}{4} \bar{M}_{2}^{(0)}\right]=\frac{3}{2} \partial_{\psi}\left(D(\psi) \partial_{\psi} \bar{P}^{(0)}\right) \\
\frac{\partial \bar{\Theta}}{\partial t}+[\phi, \bar{\Theta}]+\left[\bar{\triangle} \phi, \frac{\omega_{d 0}}{4} \bar{M}_{2}^{(1)} \psi\right] \\
+\partial_{\alpha}\left(\frac{3}{2} \omega_{d 0} \bar{P}^{(1)} \phi+\bar{M}_{2}^{(2)} \omega_{d 0}^{2} \psi+\frac{1}{4} \omega_{d 0} \bar{\triangle} \phi \bar{M}_{2}^{(1)}\right) \\
+\phi \frac{d \bar{n}}{d t}+\phi\left[\triangle \phi, \frac{3}{8} \bar{P}^{(0)}\right]-\bar{\triangle} \phi \partial_{\alpha}\left(\frac{1}{4} \omega_{d 0} \bar{M}_{2}^{(1)}\right)=\text { losses }
\end{gathered}
$$

$$
\text { losses }=\frac{3}{2} \psi \omega_{d 0} \partial_{\psi}\left(D(\psi) \partial_{\psi} \bar{P}^{(1)}\right)+\phi \partial_{\psi}\left(D(\psi) \partial_{\psi} \bar{n}\right) .
$$

In Eqs. (19)-(22), the quantities $\bar{\Theta}$ and $\bar{M}_{2}^{(1)}$ are, respectively, defined by relations (13) and (14) by substituting $f_{\kappa, E}$ by $\bar{f}_{\kappa, E}=J_{0} f_{\kappa, E}$. The smoothed ion density $\bar{n}$ and ion pressure $\bar{P}^{(n)}$ are defined by Eqs. (8) and (9) using the same substitution. A similar procedure can be used to determine the evolution equation of the kinetic density energy (21). By noting from (4) that $\bar{n}=n_{0}+\delta \phi-C_{i} \bar{\triangle} \phi-\frac{3}{8} \bar{\triangle} \bar{P}$ and by substituting the obtained relation in (21), we obtain the following relation:

$$
\begin{aligned}
& \frac{\partial \bar{\Theta}}{\partial t}+\partial_{\alpha}\left(\frac{3}{2} \omega_{d 0} \bar{P}^{(1)} \phi+\omega_{d 0}^{2} \bar{M}_{2}^{(2)} \psi+\frac{1}{4} \omega_{d 0} \bar{\triangle} \phi \bar{M}_{2}^{(1)}\right) \\
& +\left[\bar{\triangle} \phi, \frac{1}{4} \omega_{d 0} \bar{M}_{2}^{(1)} \psi\right]+\frac{3}{8} \phi[\bar{\triangle} \phi, \bar{P}] \\
& +\phi \frac{d}{d t}\left(C_{e} \delta \phi-C_{i} \bar{\triangle} \phi\right)-\frac{1}{4} \bar{\triangle} \phi \\
& \quad \times\left(\frac{3}{2} \frac{d \bar{P}^{(0)}}{d t}+\partial_{\alpha}\left(\omega_{d 0} \bar{M}_{2}^{(1)}\right)\right)=\text { losses. }
\end{aligned}
$$

The integration over $\alpha$ and $\psi$ leads to the energy conservation law (15), with however a modification of the dissipation term, which contains now an additional term due to the gyroaverage operator $J_{0}$. Using the same previous definitions (16)-(18), we have now the relation

$$
\begin{aligned}
\frac{d E_{c}}{d t}+\frac{d E_{Z F}}{d t}+\frac{d E_{\text {turb }}}{d t}= & \text { losses } \\
= & \iint \frac{d \alpha}{2 \pi} d \psi\left[\frac{3}{2} \omega_{d 0} \psi \partial_{\psi}\left(D \partial_{\psi} \bar{P}\right)\right. \\
& \left.+\phi \partial_{\psi}\left(D \partial_{\psi} \bar{n}\right)+\frac{3}{8} \bar{\triangle} \phi \partial_{\psi}\left(D \partial_{\psi} \bar{P}\right)\right]
\end{aligned}
$$

Equation (24) is obtained by using the condition (20) for the pressure $\bar{P}^{(0)}$ and we remark that, using (5),

$$
\begin{aligned}
\left\langle\left[\bar{\triangle} \phi, \bar{M}_{2}^{(0)}\right]\right\rangle_{\alpha}= & -\rho_{s}^{2} \partial_{\psi}\left\langle\delta \bar{M}_{2}^{(0)} \frac{\partial^{3} \delta \phi}{\partial \alpha^{3}}\right\rangle_{\alpha} \\
& -\delta_{b}^{2}\left\langle\delta \bar{M}_{2}^{(1)} \frac{\partial^{3} \delta \phi}{\partial \alpha \partial \psi^{2}}\right\rangle_{\alpha},
\end{aligned}
$$

where we have used the condition

$$
\bar{M}_{2}^{(0)}=\left\langle\bar{M}_{2}^{(0)}\right\rangle_{\alpha}+\delta \bar{M}_{2}^{(0)} .
$$

\section{Kinetic aspect of ZF induced by the interchange turbulence}

It is possible to introduce a "mean" pressure in the form $\bar{P}=\langle\bar{P}\rangle_{\alpha}+\delta \bar{P}$, which plays a major role, in a similar way to the zonal flow component $\langle\phi\rangle_{\alpha}$. Indeed, Eq. (20), dropping out the exponent notation $(0)$, or $\bar{P}^{(0)} \rightarrow \bar{P}$ to simplify the notation writes, after an integration over the angle $\alpha$ and considering that $J_{0} \rightarrow 1$,

$$
\begin{aligned}
\frac{\partial\langle P\rangle_{\alpha}}{\partial t}= & -\langle[\delta \phi, \delta P]\rangle_{\alpha}-\frac{2}{3} \partial_{\psi}\left\langle\left[\bar{\triangle} \delta \phi, \partial_{\alpha} \delta M_{2}\right]\right\rangle_{\alpha} \\
& +\partial_{\psi}\left(D(\psi) \partial_{\psi}\langle P\rangle_{\alpha}\right)
\end{aligned}
$$

indicating that it is the non-adiabaticity between $\delta P$ and $\delta \phi$, which drives nonlinearly a modification of the mean pressure $\langle P\rangle_{\alpha}$. Thus, for $J_{0} \rightarrow 1$ and considering $\delta_{b} \ll 1$, Eq. (27) reduces to

$$
\frac{\partial\langle P\rangle_{\alpha}}{\partial t} \simeq-\langle[\delta \phi, \delta P]\rangle_{\alpha}+\partial_{\psi}\left(D(\psi) \partial_{\psi}\langle P\rangle_{\alpha}\right) .
$$

An important question that then arises is how Eq. (27) affects the properties of zonal flows. In tokamaks, most of microturbulence is driven by ITG or ETG modes or by TIMs or TEMs. As the underlying micro-turbulence can also be driven by gradients in pressure, these constitute the energy source for the poloidal flow. The most prominent type of long wavelength toroidal micro-instability is TIM in the presence of a significant ion temperature gradient. It is wellknown that the generation of $\mathrm{ZF}$ and its feedback to driftwave turbulence are essentially nonlinear processes. ZF is generated by the Reynolds stress and back reacts upon turbulence via vortex shearing. However, it is possible to connect the time variation of $\langle\phi\rangle_{\alpha}$ of $\mathrm{ZF}$ to $\delta P$ fluctuations, driven by the interchange-type turbulence, or as we will see later in Paper II ${ }^{38}$ by resonant wave-particle interactions. Thus, the nature of ZF can be modified and its evolution is now described by 


$$
\begin{aligned}
\frac{\partial\langle\phi\rangle_{\alpha}}{\partial t}= & \left\langle\frac{\partial \delta \phi}{\partial \psi} \frac{\partial \delta \phi}{\partial \alpha}\right\rangle_{\alpha}-\frac{3}{8 C_{i}}\left\langle\frac{\partial \delta P}{\partial \psi} \frac{\partial \delta \phi}{\partial \alpha}+\frac{\partial \delta P}{\partial \alpha} \frac{\partial \delta \phi}{\partial \psi}\right\rangle_{\alpha} \\
& +\frac{1}{4 C_{i}} \partial_{\psi}\langle\delta Q\rangle_{\alpha}
\end{aligned}
$$

assuming that there is no dissipation (i.e., $D \rightarrow 0$ ) and where

$$
\langle\delta Q\rangle_{\alpha}=\int_{0}^{2 \pi} \frac{d \alpha}{2 \pi} \frac{3}{2} \delta P\left(-\frac{\partial \delta \phi}{\partial \alpha}\right) .
$$

In Eq. (29), the first term in the second member denotes the Reynolds tensor (indeed the double value), while the two following terms are a straightforward consequence of the turbulence induced by the interchange instability. The third term on the rhs of Eq. (29) is linked to the heat flux $\langle\delta Q\rangle_{\alpha}$ defined by Eq. (30). Details of the calculation are given in the Appendix.

\section{Low-frequency ZFs in the core/edge transient regime}

Analyses in the collisionless regime are required in order to clarify the characteristics of GAM-like ZF mode observed in Refs. 2 and 3 in the lower frequency range. There is a certain possibility that a new GAM-like branch (corresponding to the oscillating ZF observed at very low frequency of $4 \mathrm{kHz}$ ) in the collisionless (banana) regime where trapped particles play a major role in the interaction. Such expected waves can be observed near the critical (temperature) gradient regime and particularly for the $\mathrm{L}$ to $\mathrm{H}$-mode transition. The magnetic trapping effects contribute to give a kinetic aspect to such low-frequency GAM-type ZFs making the (modified) ZF subject to weak Landau damping. GAMs are electrostatic modes generated by the effect of the geodesic curvature, which acts linearly on the equilibrium, coupling the zero poloidal number to the (expected) sidebands $m= \pm 1$ (note that here the coupling is possible with the sidebands $n= \pm 1$ in toroidal number for the ZF component). GAMs appear as a high-frequency modulation on the top of the low-frequency zonal flow, and physically GAM can be thought of as the ion acoustic wave coupled to the geometry of the tokamak's magnetic field. The GAM dynamics strongly depends on the magnetic safety factor so that GAMs are preferentially excited in the high-q edge region, whereas the zero-frequency ZF (the expected hydrodynamical component) dominates in low q (core) region. However, in recent tokamak experiments as indicated in Refs. 2 and 3, GAMs do not appear to be active in the considered (H mode) regime, indicating Landau damping of high-frequency GAMs is strong.

However, it has been recently found that GAMs can be excited in a kinetic way by populations of fast ions in Ref. 27, the so-called high-frequency EGAM mode, showing a new kinetic aspect of GAMs. Similarly, it becomes possible to consider the case of downward frequency shift, e.g., the influence of trapped ions through the resonance with the precession frequency of thermal (trapped) ions. Moreover, the resulting low-frequency ZF is not strongly subject to Landau damping due to the strong magnetic trapping. However, hitherto, a complete picture of the dynamic interaction among GAMs, zero-frequency ZFs, and low-frequency CTIM-induced ZFs in the LH transition has not yet been achieved. Direct experimental observations of low-frequency oscillating ZFs are expected to be arduous in the core plasma because of the simultaneous presence of fluid-type ZFs (induced purely by the Reynolds stress). Thus, we forecast the situation is easier in the edge region where (zero frequency) ZF is decreasing and particularly in the H-mode regime where GAMs are strongly Landau damped.

\section{NUMERICAL SIMULATION OF COLLISIONLESS TIMs IN THE INTERCHANGE REGIME}

To determine the nature's changes of ZFs induced by TIM-KH coupling, we first study the simplest limit where only TIMs are excited.

\section{A. Linear analysis}

It is interesting to recall some general properties of the linear analysis of TIMs. In order to provide a simple physical picture of the TIM turbulence in the nonlinear regime, the dispersion relation was obtained in Ref. 24 by linearizing Eqs. (3) and (4) for an equilibrium distribution $F_{0}(\psi)$ of type (the energy $E$ being normalized to $T_{0}$ )

$$
\begin{gathered}
F_{0}(\psi)=e^{-E}\left(1+\omega_{d 0} \bar{\omega}_{d}(\kappa ; s)\left(E-\frac{3}{2}\right) \triangle \tau \psi\right) \text { with } \\
\phi_{0}(\psi)=\frac{3}{2} \omega_{d 0} \psi .
\end{gathered}
$$

In Eq. (31), the quantity $\phi_{0}$ denotes the initial flow corresponding to the interchange case. Here, $\Delta \tau$ is the normalized ion temperature gradient $\frac{\Delta \psi}{T_{0}} \frac{d T_{0}}{d \psi}$. The idea is that we want to arrive at a homogeneous set of linear equations, which will lead us to a dispersion relation for the eigenmodes of the plasma. Thus, by considering the potential perturbation mode in the form $\delta \phi_{n}(\psi) e^{i(n \alpha-\omega t)}$, one obtains the following condition (assuming the polarization term negligible in first approximation):

$C_{e} \delta \phi_{n}=\frac{2}{\sqrt{\pi}} \int_{0}^{+\infty} d E \sqrt{E} \int_{0}^{1} d \kappa \kappa K(\kappa) \frac{n \triangle \tau e^{-E}\left(E-\frac{3}{2}\right)}{E-\frac{\omega}{n \bar{\omega}_{d}(\kappa)}} J_{0} \delta \phi_{n}$

with the usual Landau prescription on the imaginary part of $\omega$. In the linear regime, the imaginary part of Eq. (32) must cancel exactly for the marginal solution, which implies that the dispersion relation for TIMs is approximated by $\omega_{\text {CTIM }}=\frac{3}{2} n \omega_{d}(\kappa)$ or equivalently for a mean value of

$$
\bar{\omega}_{C T I M} \simeq \frac{3}{2} n \omega_{d 0} \int_{0}^{1} d \kappa \kappa K(\kappa) \bar{\omega}_{d}(\kappa ; s) .
$$

As in the Landau method for electrostatic electron waves, the eigenmodes of a (collisionless) trapped ion plasma are determined by the poles of the integrand of Eq. (32). It is then possible to recover the marginal solution by solving Eq. (32). For zero boundary conditions, we obtain $\delta \phi_{n}(\psi)=$ 
$\sin l \pi \psi$ with $l=1,2,3, \ldots$. Here, a remark must be pointed out. As first indicated in Ref. 28, a better convergence of the expansion for the pole's calculation can be obtained by introducing a development around $\omega-\frac{5}{2} n \omega_{d}$ leading to an estimation of collisionless TIM frequency in the following form:

$$
\omega_{C T I M}=\frac{5}{2} n \omega_{d}(\kappa)+\frac{\sqrt{2 \varepsilon} \omega_{*}}{2(1+\tau) \rho_{s}^{2} k_{\psi}^{2}},
$$

where $\omega_{*}<0$ is the electron diamagnetic frequency. Here, the last term in the right-hand side of the equation (34) is close to $n \omega_{d}(\kappa)$.

\section{B. Numerical results in the interchange regime}

The starting point for an investigation of interchange turbulence is the reduced gyrokinetic Vlasov equation (3) coupled with the quasi-neutrality equation condition (4) initiated in an equilibrium state with a perturbation term of type

$$
\begin{aligned}
\bar{f}_{\kappa, E}(\psi, \alpha, t=0)= & F_{0}(\psi)+\phi_{\text {pert }} e^{-E} \Delta \tau \\
& \times\left(1-\frac{\pi^{2} \delta_{b}^{2} E}{4}\right) \sin (\pi \psi) \cos 5 \alpha
\end{aligned}
$$

The reason for introducing the $\sin (\pi \psi)$ factor in Eq. (35) on the right-hand side, instead of the standard potential perturbation in $\alpha$, is that this function is indeed the marginal solution obtained in the linear analysis of the CTIMs. The knowledge of the marginal solution allows us to start the ITG instability at a very low level, allowing an accurate use of our numerical scheme chosen here to integrate the system of Eqs. (3) and (4). We have adopted here a semi-Lagrangian scheme (see Refs. 29-31 for more details) to integrate the Vlasov-type equation (3), for each class of solution determined by the choice of adiabatic invariants $\kappa$ and $E$, which traces back the trajectories at every time step.

In numerical simulations, normalized quantities were used: the time is normalized to the inverse drift frequency $\omega_{d 0}^{-1}$ and the poloidal flux $\psi$ is given in $\Delta \psi$ units (with $\left.\omega_{d 0}=\frac{q_{0} T_{0}}{e B_{0} r_{0} R_{0}}\right)$. The electric potential is expressed in $\omega_{d 0} \Delta \psi$ units and the constants $C_{e}$ and $C_{i}$ introduced in the quasineutrality equation (4) are given by the relations $C_{e}=$ $\frac{T_{i}}{T_{e} f_{p}} \frac{e \omega_{d 0} \Delta \psi}{T_{0}}$ and $C_{i}=\frac{e \omega_{d 0} \Delta \psi}{T_{0}}$. The bounce and drift frequencies $\omega_{b}$ and $\omega_{d}$ depend explicitly of the pitch angle parameter $\kappa$ (and of course of the energy $E$ ) and are given by Eqs. (1) and (2).

A first simulation was carried out with an ion temperature gradient of $\triangle \tau=0.15$ chosen above the threshold of the ITG instability given by $\triangle \tau_{s}=\frac{C_{e}}{1-\frac{3}{4} \delta_{b}^{2}+\frac{15}{64} \delta_{b}^{4}} \simeq 0.100753$ for $C_{e}=0.10$ and a banana width of $\frac{\delta_{b}}{\Delta \psi}=0.10$. We choose a Larmor radius of $\frac{\rho_{s}}{\Delta \psi}=0.02$ and a magnetic shear of $s=\frac{r_{0}}{q_{0}}\left(\frac{d q}{d r}\right)_{0}=2$, well inside the strong region of the ITG instability. The phase space sampling is $N_{\psi}=256$ by $N_{\alpha}=$ 1024 and we have used $N_{\kappa} N_{E}=16 \times 128$ values in pitchangle and energy. The time step is $\triangle t \omega_{d 0}=0.001$ and we have chosen $C_{i}=1$ for the polarization term in order to excite a strong coupling with the shear flow in agreement with the analytic solution of the Taylor-Goldstein equation solved in Paper II. ${ }^{38}$ Without dissipation, three energetic subsystems interact to produce the complexity observed in the interchange-type turbulence: the kinetic energy of plasma $E_{c}$, the energy of the zonal flow noted here $E_{Z F}$, and the potential fluctuation of turbulence $E_{\text {turb }}$ contained in the initial pressure gradient. These quantities are defined by Eqs. (16), (17), and (18) and verified by the conservation law (24).

The time evolution of the energy of zonal flow $\left(E_{Z F}\right)$ is shown on the top panel in Fig. 1 in solid line, while the corresponding turbulent contribution $E_{\text {turb }}$ in energy is added on the same plot in thick line.

We have also represented, in the bottom panel, both quantities on a logarithmic scale, together with their mutual sum (the potential energy). The different components of the energy, for the same simulation, have been plotted in Fig. 2 on a logarithmic scale: the kinetic energy $\mathrm{E}_{\mathrm{c}}$, the dissipative part $E_{\text {diss }}$ together with the two components of the potential energy, $\mathrm{E}_{\mathrm{ZF}}$ (for $\mathrm{ZF}$ ) and $\mathrm{E}_{\mathrm{turb}}$, for the turbulent contribution. The total energy is also plotted in solid line. We clearly observe that the dynamics of trapped ions, through their kinetic energy contribution, exhibit low-frequency oscillations.

The dynamics of the system is first governed by the growth of TIMs, as a result of the ITG instability (in the interchange regime). Here, it is the gradient in temperature that constitutes the energy source for the growth of
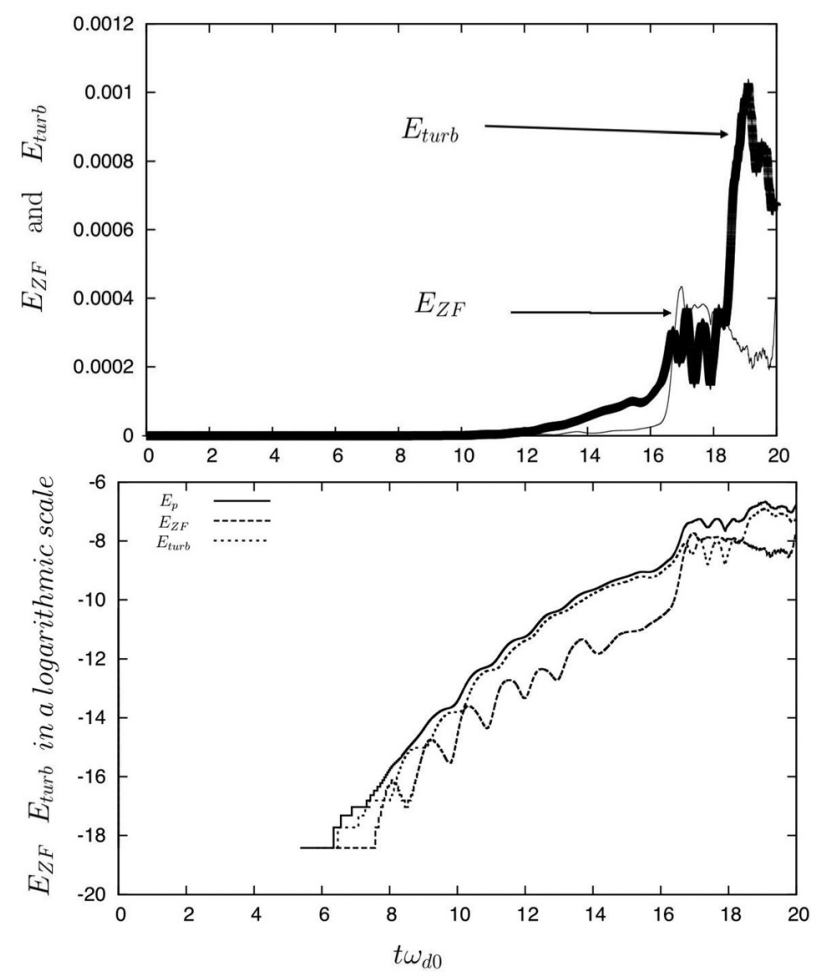

FIG. 1. On the top panel: Time evolution of the energy of the zonal flow $\left(E_{Z F}\right)$ in solid line and of the corresponding turbulent energy $E_{\text {turb }}$ in thick line in the regime of the interchange instability. We observe a turbulent burst for $t \omega_{d 0}>18$ accompanied with the decrease of the zonal flow energy. In the bottom panel: corresponding evolution, in a logarithmic scale, of the zonal flow $E_{Z F}$ and the turbulent energy $E_{\text {turb }}$ together with their mutual sum. 


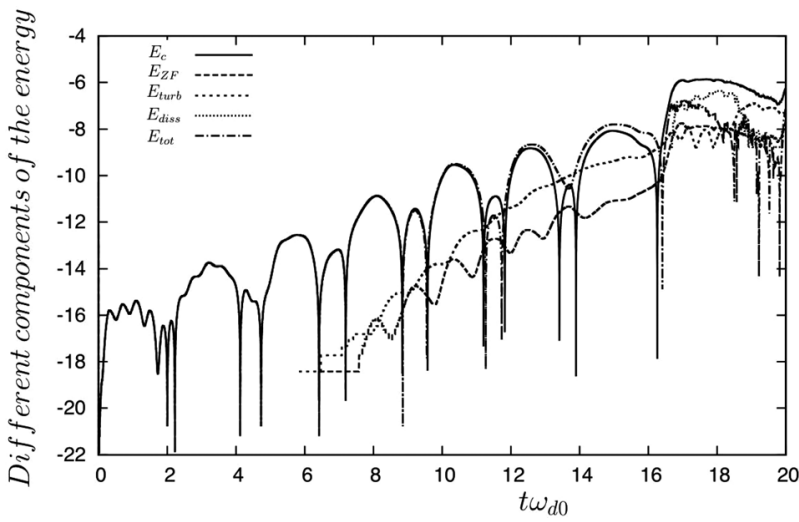

FIG. 2. Time evolution of the different energy components: the zonal flow $E_{Z F}$, the turbulent part $E_{\text {turb }}$, the kinetic energy $E_{c}$, the dissipative part $E_{\text {diss }}$, together with the total energy plotted in solid line. The different contributions are plotted in a logarithmic scale.

(collisionless) TIMs. Since we have perturbed initially the mode $n=5$, we expect the occurring of five vortices in phase space, as can be seen in Fig. 4 on the top panel at time $t \omega_{d 0}=0.20$. However, the mode $n=1$ becomes dominant and seems to play a major role before the saturation of the instability takes place. In addition to the growth of CTIMs, the nonlinear interaction leads to the growth of a time-varying zonal flow (corresponding in the trapped-ion model to the mode $n=0$ ), followed by a strong increase of the turbulent activity associated with the formation of streamers. The time evolution of both dominant modes $n=0$ (zonal flow) and $n=1$ (resonant CTIM) is shown in Fig. 3 in a logarithmic scale. The maximum value of the growth rate for the dominant
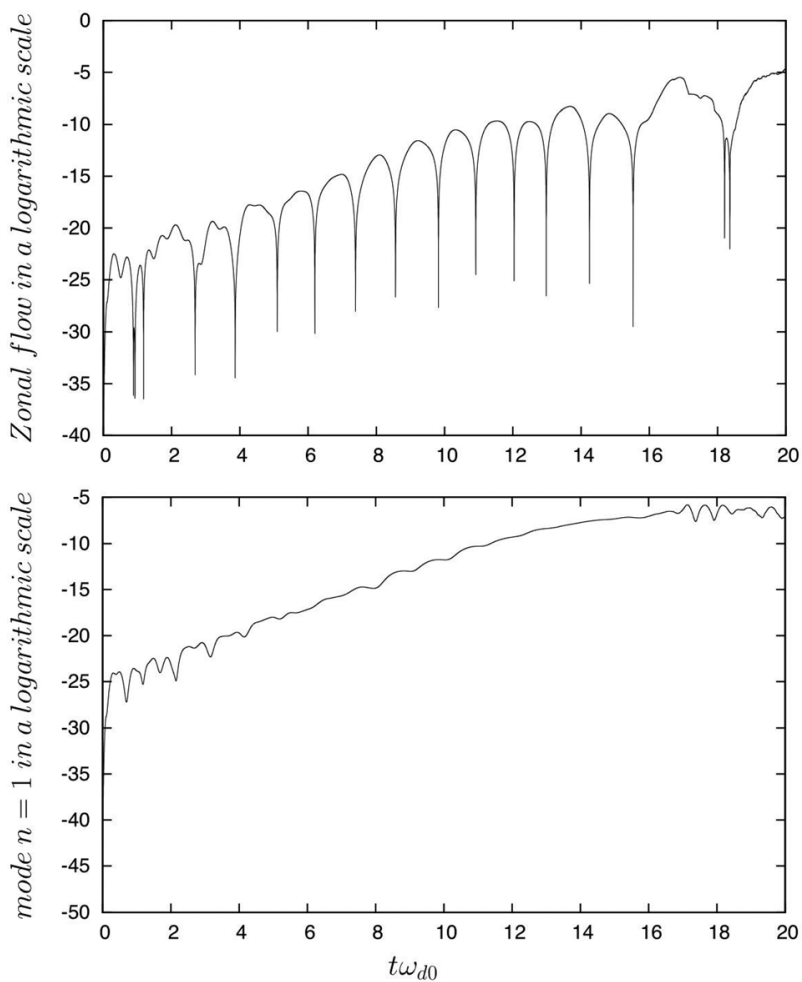

FIG. 3. Time evolution of the flow (mode $n=0$ ) in a logarithmic scale: the zonal flow exhibits an oscillatory behaviour linked to the pressure contribution of resonant trapped-ion mode. In the bottom panel, the resonant mode. The simulation was performed in the TIM regime. mode $n=1$ is found close to $\frac{\gamma}{\omega_{d 0}} \simeq 0.670$, a numerical value somewhat higher than the linear expected (interchange) value given by $\frac{\gamma_{t h}}{\omega_{d 0}}=\sqrt{\frac{3}{2}} \frac{\sqrt{\Delta \tau-\Delta \tau_{\text {threshold }}}}{\sqrt{C_{i} \delta_{b}^{2} l^{2} \pi^{2}+C_{e}+C_{i} n^{2} \rho_{s}^{2}}} \simeq 0.610$.

The time-varying ZF generation mechanism is a nonlinear process in which shorter-scale fluctuations transfer their energy to larger-scale potential structures. Due to the interchange instability, the resulting effect on the pressure may lead to a trapped-ion version of a basic mode of oscillation involving the contribution of a resonant trapped-ion mode in the collisionless regime via the pressure term. Note that such pressure effects are usually invoked in the generation of high-frequency GAM in Refs. 32-35. The collisional damping of GAMs is studied in Ref. 36. It must be pointed out that, in our case, it is not the coupling with the acoustic branch (which is usually observed in GAMs), which takes place but the interaction with the resonant version of TIM, which differs for the usual fluid-type interchange mode (see Ref. 28). In fact in Paper II, ${ }^{38}$ we will show that both interchange and resonant counterpart CTIM coexist and can beat in a three-wave interaction leading to the (non zero) lowfrequency $\mathrm{ZF}$ growth. The analysis of the top panel in Fig. 3 shows, however, that the zonal flow exhibits a real frequency close to $\omega \simeq 2.7 \omega_{d 0}$, i.e., a value well below the initially excited CTIM frequency of $\omega_{T I M}=\frac{5}{2} n\left\langle\bar{\omega}_{d}(\kappa ; s)\right\rangle_{\kappa} \simeq$ $26.39 \omega_{d 0}$ for the mode $n=5$. Note that the observed frequency is close to the frequency of the resonant mode for $n=1$ found close to $\omega_{\text {resTIM }} \simeq \frac{3}{2} n\left\langle\bar{\omega}_{d}(\kappa ; s)\right\rangle_{\kappa} \simeq 3.16 \omega_{d 0}$, using a magnetic shear of $s=2$ and $\left\langle\bar{\omega}_{d}\right\rangle_{\kappa} \simeq 2.11$.

To maintain the connection with the EAST tokamak experiment presented in Ref. 2, we have estimated, for the toroidal number $\mathrm{n}=1$, the value of the oscillation frequency of the ZF close to

$$
\begin{aligned}
f & \simeq \frac{2.7 \omega_{d 0}}{2 \pi}=2.7 \frac{q^{3}}{2 \pi \omega_{c i} \varepsilon}\left(\frac{v_{t h i}}{q R_{0}}\right) \sim 2.7 \times 0.68 \mathrm{kHz} \\
& =1.84 \mathrm{kHz} .
\end{aligned}
$$

The physical parameters are $r_{0}=0.45 \mathrm{~m}, R_{0}=1.88 \mathrm{~m}$, $B_{0} \sim 1 T$ (corresponding to a value of the ion cyclotron frequency of $\omega_{c i} \sim 10^{8} \mathrm{~Hz}$ ) for $\mathrm{q}=4$ and an aspect ratio of $\varepsilon=\frac{r_{0}}{R_{0}}=0.239$. The ion transit frequency is chosen close to $\frac{v_{t h i}}{q R_{0}} \sim 2 \times 10^{4} \mathrm{~Hz}$ at the plasma edge. Thus, this value is found in well agreement with the peak of $2 \mathrm{kHz}$ observed in the potential fluctuation power spectra.

It must be pointed out that the burst of turbulent activity observed in Fig. 1 for $t \omega_{d 0} \geq 18$ is accompanied by a slight decrease of the ZF energy. Such a behaviour points out the possibility that there may be a coupling between $\mathrm{ZF}$ and streamers. The whole picture of the generation of streamer and $\mathrm{ZF}$ actors is completed by analyzing the behaviour of the electric potential $\phi(\psi, \alpha, t)$ and the $\boldsymbol{v}_{E}=\frac{\boldsymbol{E} \times \boldsymbol{B}}{B^{2}}$ drift velocity, respectively, shown in Figs. 4 and 5 at three different times. As previously mentioned, since we have initially excited the toroidal mode number $n=5$, the top panel in Fig. 4 exhibits five coherent periodic structures, as expected, corresponding to the mode $n=5$. The top panel in Fig. 5 displays the 

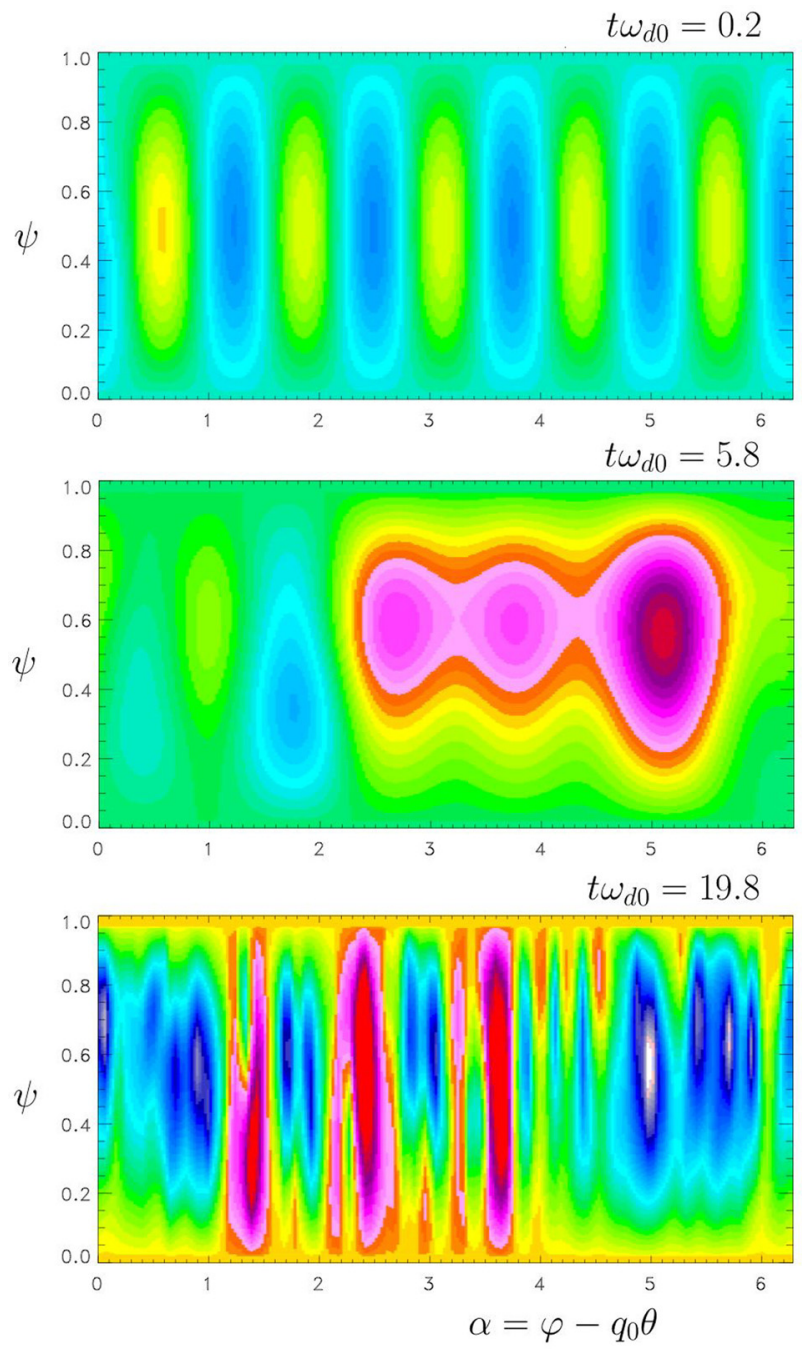

FIG. 4. Phase space $\psi, \alpha$ representation of the electric potential at three different times during the plasma evolution. We see clearly the formation of TIM on the top panel for the mode $n=5$, followed by the growth of the mode $n=1$. The bottom panel shows the excitation of nonlinear streamers during the turbulent burst. The simulation was performed in the TIM regime.

corresponding velocity shear flow indicating that the main part of the current results from the $\boldsymbol{v}_{E}$ drift velocity, which takes place along the $\psi$ direction. As sketched in middle panels in Figs. 4 and 5, the nature and topology of the potential change fundamentally show now a strong modulation of the electric potential on the toroidal mode $n=1$ in Fig. 4. The middle panel in Fig. 5 shows a strong modification in the direction of the flow located in the region of the vortex structure of positive values of the electric potential. Finally, at $t \omega_{d 0} \simeq 20$, the bottom panels in Figs. 4 and 5 show the formation of nonlinear streamers (elongated in $\psi$ ) as the result of the re-emergence of turbulent activity observed at that time in Fig. 1. During this phase, a second topology modification takes place in the shear velocity flow when nonlinear streamers are strongly generated.

In the framework of the trapped-ion model, we have obtained in Section III C the equation of the mean potential fluctuations $\langle\phi\rangle_{\alpha}$ dynamics with respect to zonal flow $\langle\phi\rangle_{Z F, K H}$ potential and the trapped-ion interchange-type counterpart $\langle\phi\rangle_{Z F, T I M}$ as follows:
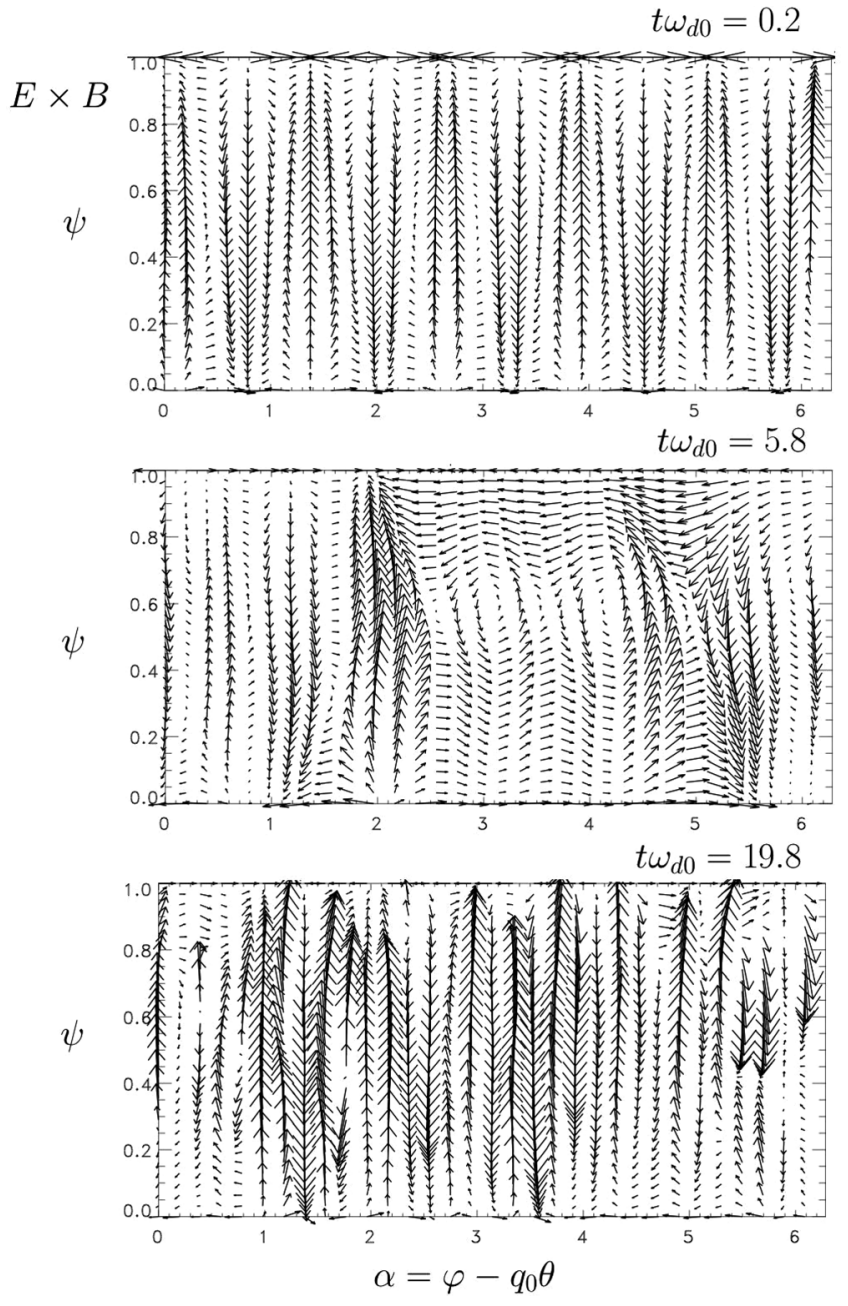

FIG. 5. Dynamics behaviour of the $\boldsymbol{E} \times \boldsymbol{B}$ shear flow velocity at different times. The topology of the electric potential is here strongly modified in the TIM regime.

$$
\begin{gathered}
\langle\phi\rangle_{\alpha}=\langle\phi\rangle_{Z F, K H}+\langle\phi\rangle_{Z F, T I M}, \\
\frac{\partial\langle\phi\rangle_{Z F, K H}}{\partial t}=\frac{1}{2}\left\langle\frac{\partial \delta \phi}{\partial \psi} \frac{\partial \delta \phi}{\partial \alpha}\right\rangle_{\alpha},
\end{gathered}
$$

$$
\begin{aligned}
\frac{\partial\langle\phi\rangle_{\text {ZF,TIM }}}{\partial t}= & \frac{1}{2}\left\langle\frac{\partial \delta \phi}{\partial \psi} \frac{\partial \delta \phi}{\partial \alpha}\right\rangle_{\alpha}-\frac{3}{8 C_{i}}\left\langle\frac{\partial \delta P}{\partial \psi} \frac{\partial \delta \phi}{\partial \alpha}+\frac{\partial \delta P}{\partial \alpha} \frac{\partial \delta \phi}{\partial \psi}\right\rangle_{\alpha} \\
& +\frac{1}{4 C_{i}} \frac{\partial}{\partial \psi}\langle\delta Q\rangle_{\alpha} .
\end{aligned}
$$

There are two basic effects in the $\langle\phi\rangle_{\alpha}$ dynamics evolution: the interchange dynamics itself in Eq. (38) and the standard turbulence-induced Reynolds stress already mentioned by Diamond and Kim. ${ }^{37}$ The nonlinear drive of zonal flows is governed by the Reynolds tensor in Eq. (37). It is balanced by the (non-adiabatic) interchange-type transfer given by (38). It is the polarization drift nonlinearly occurring in the right-hand side of Eq. (38), which gives the main contribution. It is worth pointing out at this stage that Eq. (38), described above, contains two nonlinear interaction terms. The first one behaves in exactly the opposed fashion than the Reynolds tensor when adiabatic conditions set in (since in that case $\left.\delta P=\frac{8}{3} C_{i} \delta \phi\right)$. The last term in (38) linked to the 
heat flux fluctuations $\langle\delta Q\rangle_{\alpha}$ allows a coupling with $\langle P\rangle_{\alpha}$. Averaging the gyrokinetic Vlasov equation (3) leads to the heat equation (without losses)

$$
\frac{3}{2} \frac{\partial \bar{P}}{\partial t}+\frac{\partial \bar{Q}}{\partial \psi}=0 .
$$

In the limit of adiabatic conditions where $\delta P$ is proportional to $\delta \phi$, the profile of $\langle P\rangle_{\alpha}$ is not nonlinearly modified since the right-hand side of Eq. (28) disappears. However, the second member of (38) reduces to zero.

Now, we get the entire physical picture as follows. In the initial stage, the dominant mode is the fluid-type interchange mode leading to a weak and (adiabatic) slow increase of the zonal flow. As the resonant CTIM is excited, corresponding to the mode $n=1$ with a frequency $\omega_{d}(\kappa) E=\omega_{d 0} \bar{\omega}_{d}(\kappa) E$ (the resonance takes place for $E \sim \frac{3}{2} T_{0}$ ), we observe a fast growth of the zonal flow structure linked to non adiabaticity effects induced by polarization $\left(C_{i} \neq 0\right)$ plus the average operator $J_{0}$ effect. At that time, the Reynolds tensor grows also rapidly due to resonance. At time $t \omega_{d 0}=18$, the burst in turbulence is associated with the formation of nonlinear small-scale streamers, which corresponds to the observed decrease in the ZF energy.

Fig. 6 shows the profile in pressure on the top panel and of the density in the bottom panel, respectively, at time $t \omega_{d 0}=16$, showing the beginning of the profile modification driven by both polarization and resonance effects. Fig. 7 portrays the state of the plasma at that time. All the plots show
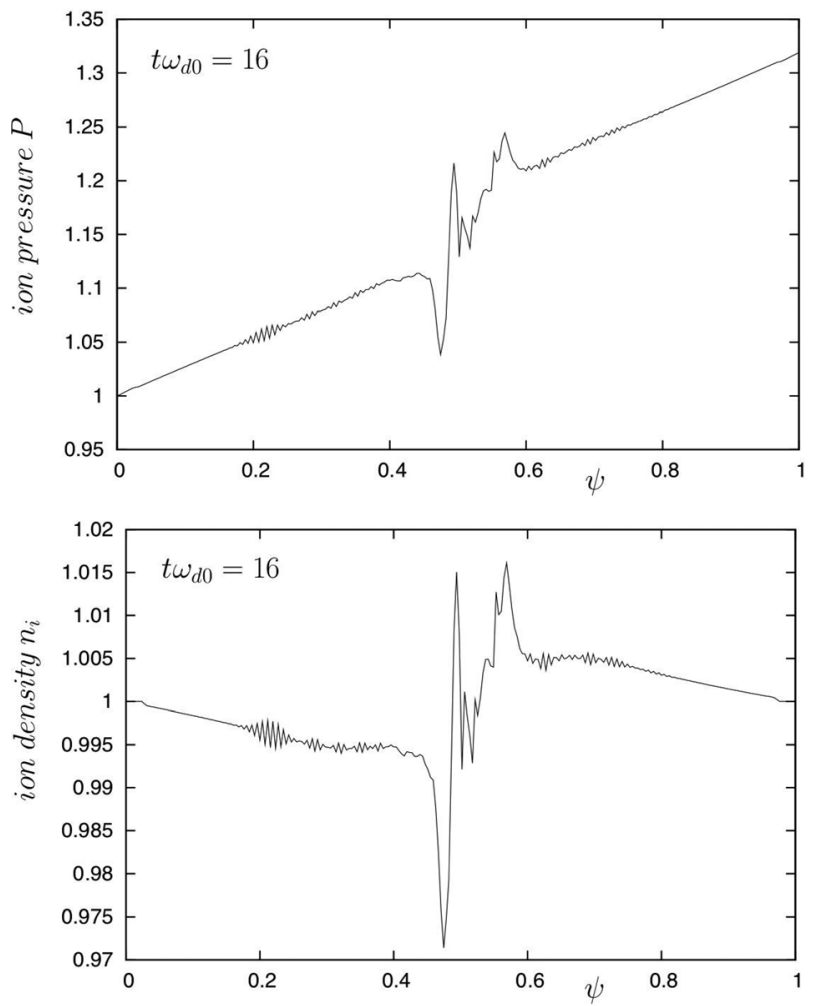

FIG. 6. Ion pressure (on the top) and ion density (in the bottom) profiles observed in simulation at time $t \omega_{d 0}=16$ at the beginning of the growth of the turbulent energy.
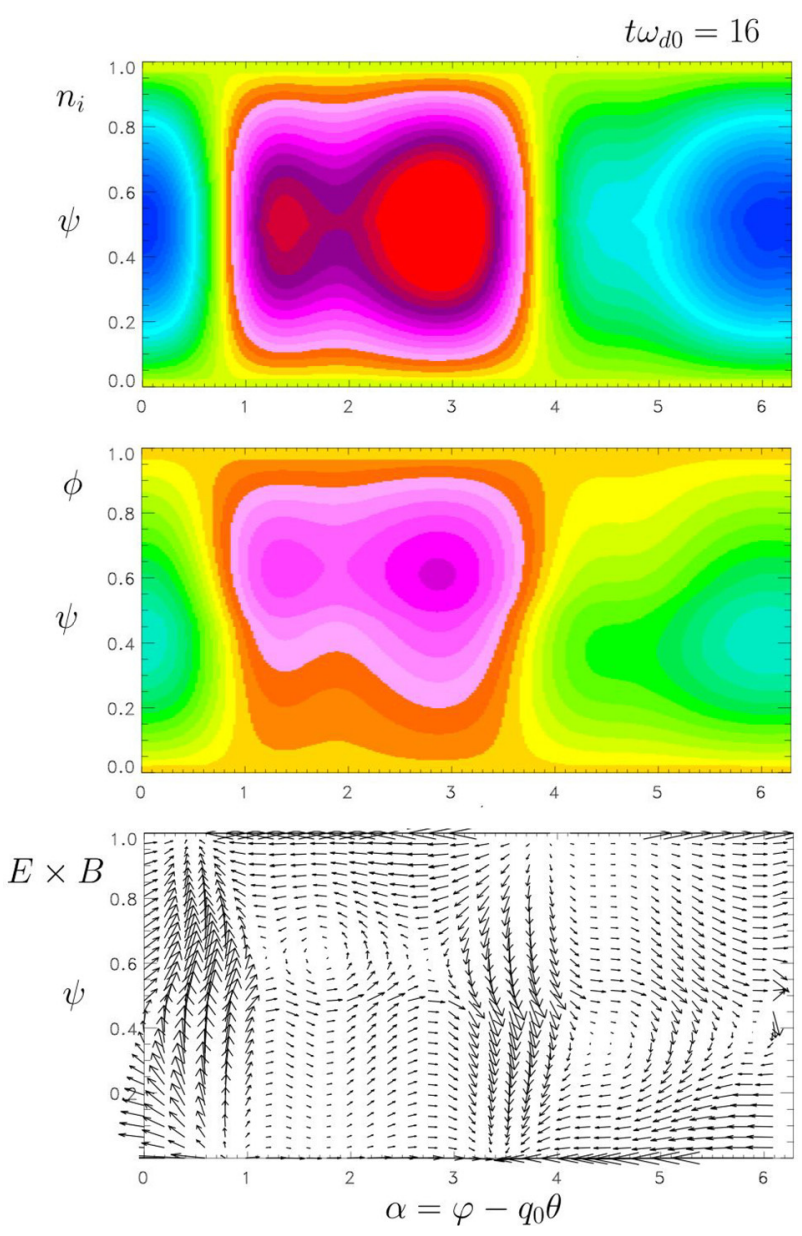

FIG. 7. Behaviour in phase space of the ion density (on the top panel) of the corresponding electric potential (on middle panel) and of the $\boldsymbol{E} \times \boldsymbol{B}$ drift velocity (in the bottom panel) at the same time $t \omega_{d 0}=16$ at the resonance. We see clearly that both polarization effects and change in the shear velocity direction play a major role.

the formation of a long-scale structure (corresponding to the toroidal number $n=1$ ). This state is seen to begin to be non adiabatic (density and electric potential start to differ). It is also interesting to note that the low frequency of ZF is modified, which seems to indicate that the resonance of TIM with precession drifts leads to coupling with $\mathrm{KH}$ modes. This is illustrated by the plot shown in the bottom panel in Fig. 7. Our present study is confined to an interchange plus resonant case to highlight the role of the trapped-ion resonance on $\mathrm{ZF}$ generation in the pattern of interchange turbulence.

\section{NUMERICAL RESULTS IN THE KH REGIME}

In this section, we study a simple example with the $\mathrm{KH}$ instability to elucidate some of the key features of the nature of the associated $\mathrm{ZF}$ in the simplest context of coupling with the interchange turbulence. ZF formation is commonly attributed to nonlinear mechanisms such as Reynolds stress. Other actors that play a major role are streamers. Such structures trigger an intermittent heat flux that necessarily competes against ZFs. One of the possible mechanisms of saturation of $\mathrm{ZF}$ growth is the $\mathrm{KH}$ instability of the zonal flows, driven by their shear. We examine the case of the $\mathrm{KH}$ 
instability driven by an initial velocity shear of $\phi_{0}(\psi)=$ $\phi_{\max } \frac{\sin (2 \pi \psi)}{2 \pi}$ in the case of an equilibrium distribution of type

$$
F_{0}(\psi)=e^{-E}\left[1+\left(\frac{5}{2}-E\right) 2 \pi \phi_{0}(\psi) C_{i} \delta_{b}^{2}\right] .
$$

A perturbation is introduced initially on the first five toroidal numbers in the form $e^{-E} \phi_{\text {pert }} \sin (\pi \psi) \sum_{n=1,5} \cos (n \alpha)$. Here, we use the following physical parameters in normalized units: $\phi_{\text {max }}=2.50, \phi_{\text {pert }}=10^{-4}, \quad C_{e}=0.10, \quad C_{i}=1, \quad \delta_{b}=0.10$, and $\rho_{s}=0.02$. The phase space sampling is $N_{\psi}=256$ and $N_{\alpha}=512$ and the numerical simulation has been performed again using $N_{E}=128$ times $N_{\kappa}=16$ adiabatic invariants in energy and pitch-angle. We have used a temperature gradient of $\triangle \tau=0$ to focus on the pure KH instability (at least in the initial stage). The corresponding time evolution of the $\mathrm{ZF}$ energy (in solid line) and the turbulent counterpart is plotted in Fig. 8 on the top panel in thick line, where we have also superimposed their mutual sum. We have also represented the time evolution of the mode $n=5$ of the potential energy in the bottom panel in a logarithmic scale. We see clearly that the linear regime ends when saturation is reached at time $t \omega_{d 0} \simeq 1.2$. The growth-rate observed in the time evolution of the energy of the initial mode $n=5$ is found numerically close to $\frac{\gamma}{\omega_{d 0}} \simeq 7.70$ in well agreement with the expected value of $\frac{\gamma_{t h}}{\omega_{d 0}}=\left(n_{s}-n\right) \frac{n_{s} \rho_{s}^{2}}{4 \pi^{2} \delta_{b}^{2}} \quad$ obtained using the Taylor-Goldstein
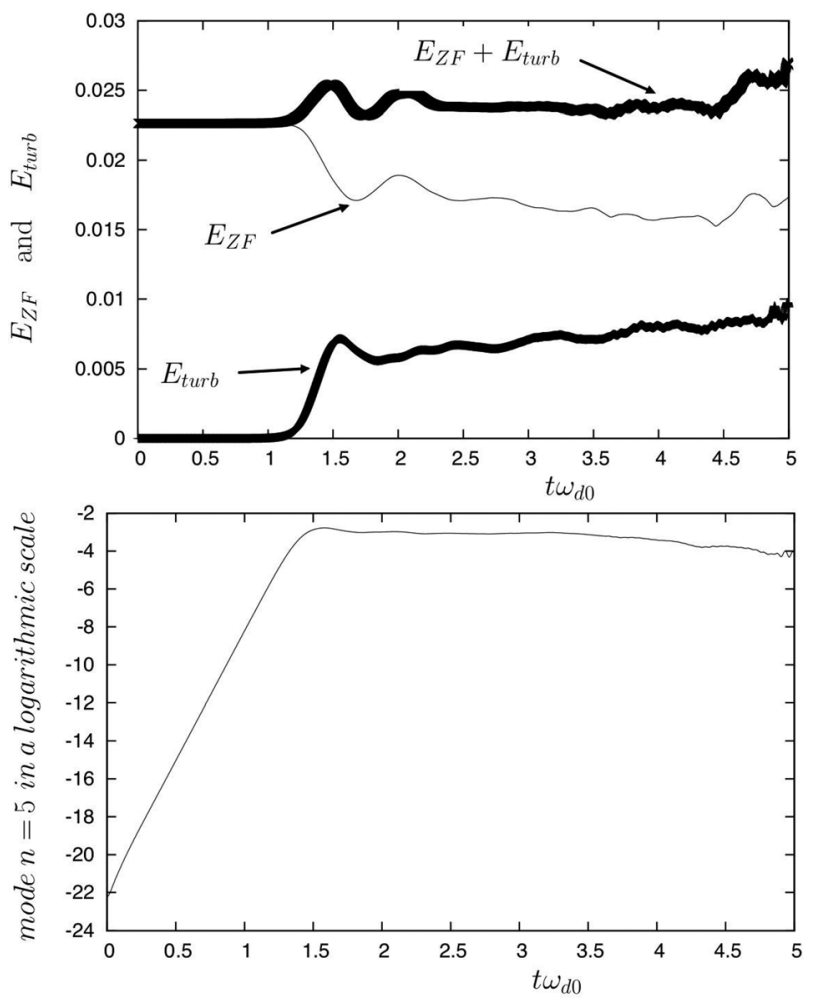

FIG. 8. On the top panel, the time evolution of the zonal flow energy (in solid line), the turbulent part (in thick line), and their mutual sum in the $\mathrm{KH}$ regime. In the bottom panel, the time evolution of the mode $n=5$, initially excited, of the potential energy, in a logarithmic scale indicated the beginning of the nonlinear phase of the instability (i.e., for $t \omega_{d 0}>1.2$ ). formulation (for more details, see Paper II), which is given by $\frac{\gamma_{t h}}{\omega_{d 0}} \simeq 7.65$.

The evolution of the $\mathrm{KH}$ instability is first characterized by a linear regime, where both energies $E_{Z F}$ and $E_{\text {turb }}$ remain quasi constant (on the top panel). First, it is clear that the observed fluctuations are triggered by nonlinear effects induced by the KH instability. Thus, the increase of the turbulent energy is linked to an energy transfer from the initial shear flow towards the turbulence. Thus, a second turbulence characteristic necessary to $\mathrm{ZF}$ decrease is some conservation from the total ZF plus turbulence energy. Fig. 9 shows the typical time evolution of the mean potential $\langle\phi\rangle_{\alpha}(\psi, t)$ on the top panel and the Reynolds tensor $\frac{1}{2}\left\langle\frac{\partial \delta \phi}{\partial \alpha} \frac{\partial \delta \phi}{\partial \psi}\right\rangle_{\alpha}$ in the bottom panel. In the beginning of the saturation regime, the Reynolds tensor is the dominant term, but it is, however, not possible to neglect the interchange contribution at later time. The top panel in Fig. 9 illustrates the details of the ZF dynamics. The underlying mechanism for zonal flow decrease in turbulence is given by Eq. (29), i.e., by the coupling between the Reynolds tensor and the interchange-type terms. The presence of both effects results in a significant reduction of the ZF energy. The effect is apparent from the early stage of the growth of turbulent energy, when nonlinear coupling begins to occur. We must stress that the resulting coupling is a highly nonlinear effect.

Figs. 10 and 11 display the electric potential and the corresponding $\boldsymbol{v}_{E}$ drift at three different times during the evolution. The shape of the large-scale structures changes from one characterized by $\mathrm{KH}$ vortices in the early stage of the

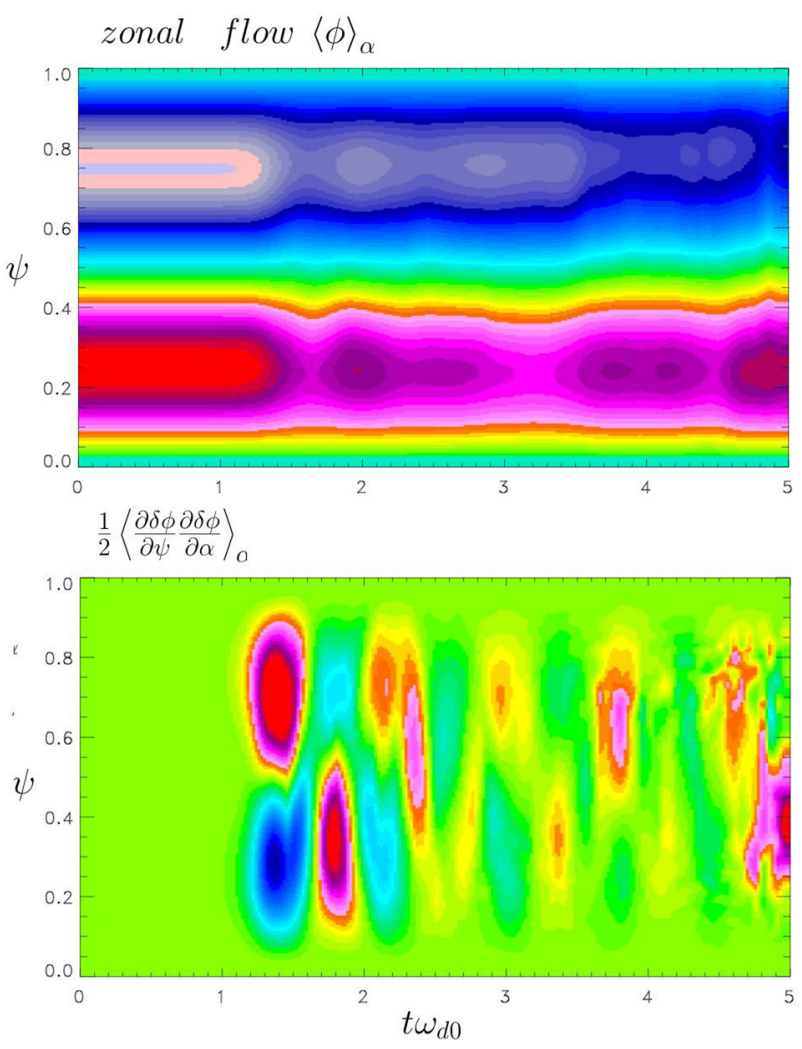

FIG. 9. Dynamical behaviour of the zonal flow in the plane $(\psi, t)$ on the top panel. We have plotted in the bottom panel the corresponding time- $\psi$ evolution of the Reynolds tensor. 

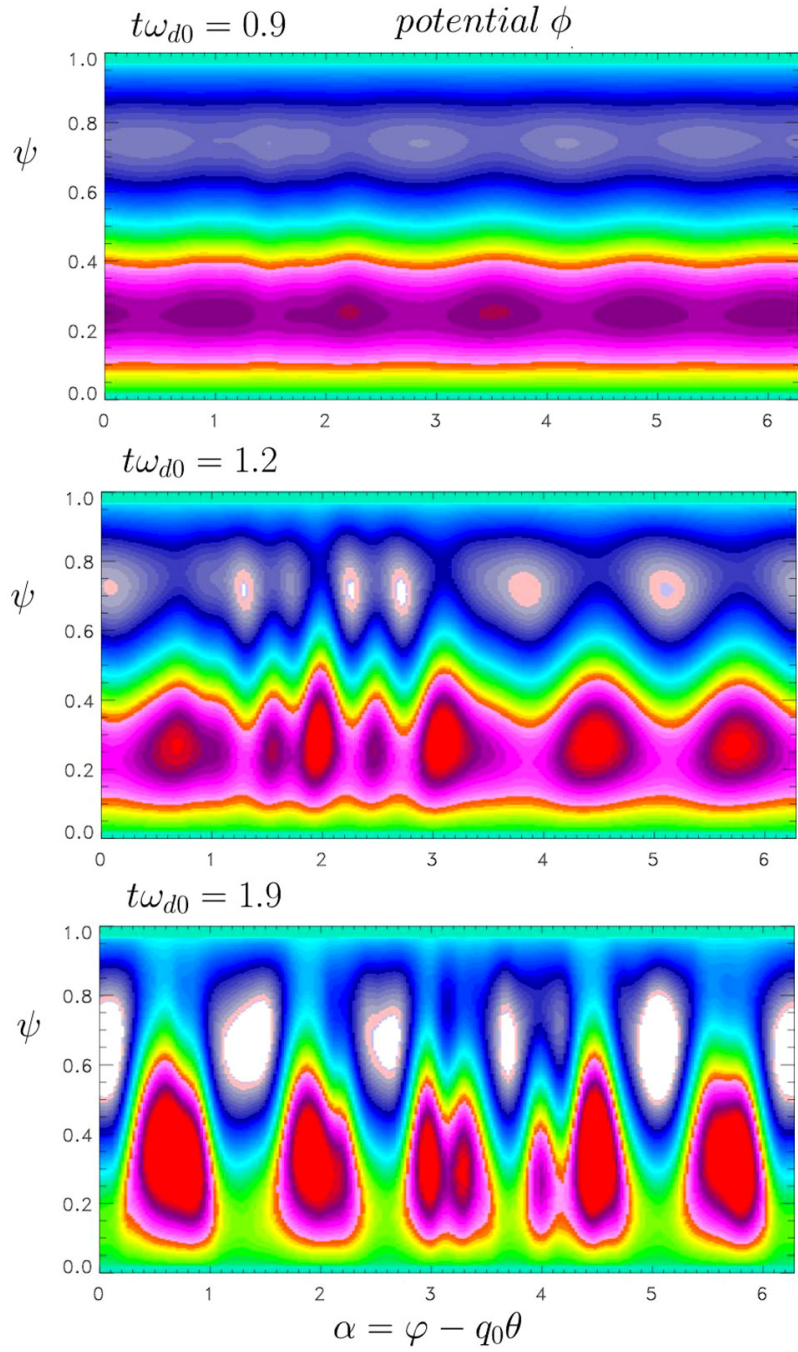

FIG. 10. Phase space plots of the electric potential at three different times: in the linear phase (on the top), at saturation (on middle), and later (in the bottom panel). The last curve exhibits a generalized $\mathrm{KH}$ structure, i.e., an initially nonlinear $\mathrm{KH}$ structure modified by the combined action of polarization effects and interchange instability. We have used $\phi_{\max }=2.5$.

evolution to more turbulent structures in the final state (referred as generalized KH vortices). Such nonlinear structures result from the formation of nonlinear $\mathrm{KH}$ vortices. Before closing the analysis, such structures correspond to strong density fluctuations that can lead to a destabilization of TIMs by modifying nonlinearly the gradient in density and temperature. While standard $\mathrm{KH}$ structures are formed on the top panel in Fig. 10, their topology is, however, modified in Fig. 11, leading to a solution in the bottom panel, which looks like the so-called generalized KH structures introduced by Kim and Diamonds. ${ }^{21}$ Physically, even when the temperature gradient is initially zero, the turbulence sets in and seems to be driven by nonlinear effects and fluctuations in density, which may also lead to the start-up of the ITG instability.

It is worth stressing that the reduction in ZF energy is enhanced when we increase the amplitude of the KH shear flow. An example is shown in Fig. 12, where we have chosen to increase $\phi_{\max }$ to the value of 5 . Other physical parameters are kept identical to those of Figs. 8 to 11. Note the strong
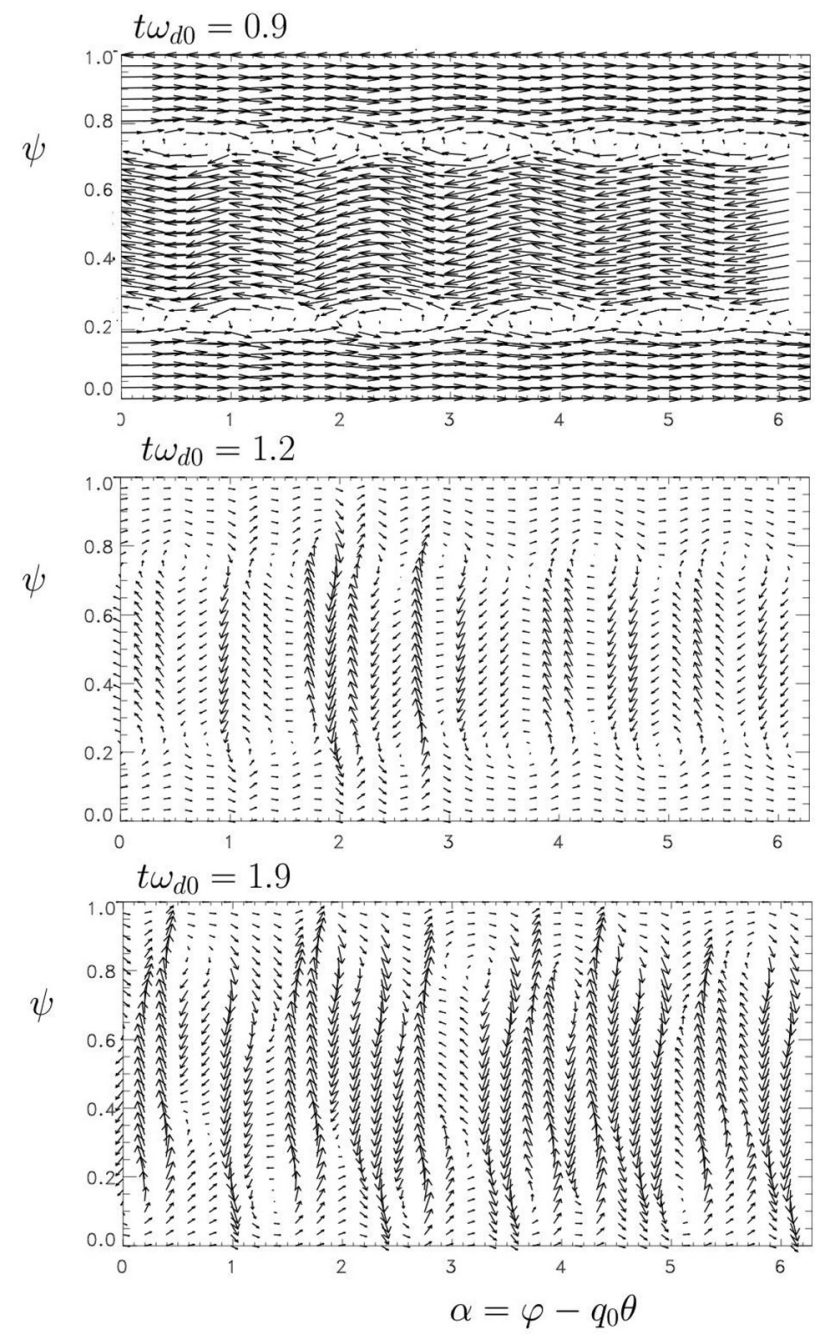

FIG. 11. The corresponding $\boldsymbol{E} \times \boldsymbol{B}$ drift-velocity showing the change in the topology of the shear flow and thus in the nature of the $\mathrm{KH}$ mode. We have used $\phi_{\max }=2.5$.

growth of the turbulent energy and the associated reduction of the ZF energy on the top panel in Fig. 12. Such nonlinear effect, not explained by linear analysis (where $\langle\phi\rangle_{\alpha}=0$ ), is systematically recovered in both types of simulations. The evolution of the kinetic energy is related with the flow structures that are illustrated by the plot of the kinetic energy in the bottom panel in Fig. 12. As can be seen clearly, there are three distinct stages. The early stage (for $t \omega_{d 0} \leq 0.5$ ) corresponds to the linear regime followed by the strong decrease of the kinetic energy, which corresponds to the energy transfer to the total energy shown in the top panel, which in turn promotes the fast growth of streamers. The last regime is linked to the slow growth of the turbulent energy.

\section{CONCLUSION}

In this work, we have studied the effects of sheared poloidal flows on ion-temperature gradient modes, in the regime of trapped-ion modes. We have identified a nonlinear coupling mechanism of zonal flow mediated by trapped-ion modes in the collisionless regime. The driving force of the zonal flow was derived, in which contributions of 

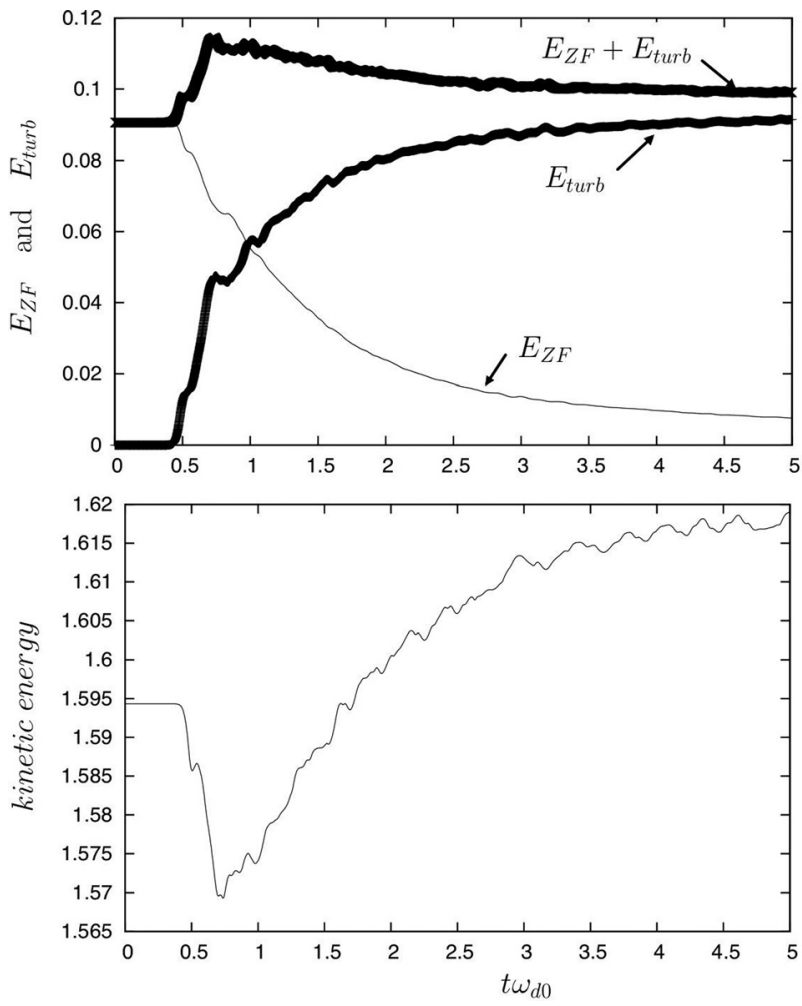

FIG. 12. On the top panel, the time evolution of the zonal flow energy, the potential energy, and their mutual sum for $\phi_{\max }=5$. Note that the sum of $E_{Z F}+E_{\text {turb }}$ is almost conserved excepted at the beginning of the nonlinear phase where streamers are also excited. The growth of streamers is accompanied of a strong decrease in kinetic energy as observed in the bottom panel.

interchange mode are included with particular emphasis when a resonant trapped ion mode is excited. The obtained formula extends the action of the famous Reynolds tensor to wider cases. This leads to a low-frequency zonal flow mode of resonant nature. Several remarks must be pointed out. Clearly, a kinetic treatment of this zonal flow regime that incorporates damping or amplification by resonant wave"banana" resonance is ultimately necessary. Of course, the trapped-ion model, with or without flux term source, is a good candidate to explore such a physics. This reduced kinetic Vlasov model and the associated Semi-Lagrangian simulations give prototypical examples to understand the mutual interaction between zonal flow, (interchange) TIMs and their resonant contribution, nonlinear $\mathrm{KH}$ modes, and nonlinear streamers.

\section{ACKNOWLEDGMENTS}

The authors are indebted to the IDRIS computational center, Orsay, France, for computer time allocation on their computers. This work was granted access to the HPC resources (Grant No. 2014-2015-054028) made by GENCI (Grand Equipement National de Calcul Intensif). This work was carried out within the framework of the European Fusion Development Agreement and the French Research Federation for Fusion studies (FR FCM). It was supported by the European Communities under the contract CfP WP14ENR-01 of Associations between EURATOM and CEA.

\section{APPENDIX: TIME EVOLUTION OF THE MEAN POTENTIAL}

In this Appendix, the time evolution of the mean zonal flow potential $\langle\phi\rangle_{\alpha}$ is derived. To simplify the presentation, we do not take into account the dissipative term in the rhs of the Vlasov equation (3). It is first convenient to apply the gyro-average operator $J_{0} \simeq 1+\frac{E}{4} \bar{\triangle}$ to the reduced Vlasov equation (3) and then to integrate over the parameters $\kappa$ and $E$ leading to

$$
\partial_{t} \overline{\bar{n}}+\frac{3}{2} \omega_{d 0} \partial_{\alpha} \overline{\bar{P}}^{(1)}=\int_{0}^{1} d \kappa \kappa K(\kappa) \frac{2}{\sqrt{\pi}} \int_{0}^{+\infty} d E \sqrt{E}\left(-J_{0}\left[J_{0} \phi, \bar{f}\right]\right)
$$

using the standard notation

$$
\begin{gathered}
\overline{\bar{n}}=\int_{0}^{1} d \kappa \kappa K(\kappa) \frac{2}{\sqrt{\pi}} \int_{0}^{+\infty} d E \sqrt{E} J_{0} \bar{f}, \\
\frac{3}{2} \overline{\bar{P}}^{(1)}=\int_{0}^{1} d \kappa \kappa K(\kappa) \frac{2}{\sqrt{\pi}} \int_{0}^{+\infty} d E \sqrt{E} E J_{0} \bar{f} .
\end{gathered}
$$

Using Eq. (4), we replace $\overline{\bar{n}}$ by the expression $n_{0}+C_{e} \delta \phi-$ $C_{i} \bar{\triangle} \phi$ and we integrate (A1) over the angle variable $\alpha$. We then obtain

$-C_{i} \partial_{t} \bar{\triangle}\langle\phi\rangle_{\alpha}=\int_{0}^{1} d \kappa \kappa K(\kappa) \frac{2}{\sqrt{\pi}} \int_{0}^{+\infty} d E \sqrt{E}\left(-\left\langle J_{0}\left[J_{0} \phi, \bar{f}\right]\right\rangle_{\alpha}\right)$,

and we have noted $\bar{f}=J_{0} f$. By writing the bracket $J_{0}\left[J_{0} \phi, \bar{f}\right]$ in the form $J_{0}\left[J_{0} \phi, \bar{f}\right]=[\bar{\phi}, \bar{f}]+\frac{\bar{\Delta}}{4}[\bar{\phi}, \bar{f}]$, a little algebra gives

$$
J_{0}\left[J_{0} \phi, \bar{f}\right]=\left[\phi, J_{0}^{2} f\right]-\frac{E}{4}[\phi, \bar{\triangle} f]+\frac{E}{4}[\bar{\triangle} \phi, f]+\frac{E}{4}[\phi, f] .
$$

By introducing the following quantities $\phi=\langle\phi\rangle_{\alpha}+\delta \phi$ and $P=\langle P\rangle_{\alpha}+\delta P$, where $\langle\delta \phi\rangle_{\alpha}=\langle\delta P\rangle_{\alpha}=0$, and by substituting (A5) into (A4) we obtain

$$
\begin{aligned}
-C_{i} \partial_{t} \bar{\triangle}\langle\phi\rangle_{\alpha}= & C_{i}\langle[\delta \phi, \bar{\triangle} \delta \phi]\rangle+\frac{3}{8}\left(\langle[\delta \phi, \bar{\triangle} \delta P]\rangle_{\alpha}\right. \\
& \left.-\langle[\bar{\triangle} \delta \phi, \delta P]\rangle_{\alpha}\right)-\frac{3}{8}\langle\bar{\triangle}[\delta \phi, \delta P]\rangle
\end{aligned}
$$

In Eq. (A6), we must determine the three different Poisson brackets in the rhs of (A6).

(i) The first term $[\phi, \bar{\triangle} \phi]=\frac{\partial \phi}{\partial \psi} \frac{\partial \bar{\Delta} \phi}{\partial \alpha}-\frac{\partial \phi}{\partial \alpha} \frac{\partial \bar{\Delta} \phi}{\partial \psi}$ writes as

$$
\begin{aligned}
{[\phi, \bar{\triangle} \phi]=} & \delta_{\mathbf{b}}^{2}\left(\partial_{\alpha} \partial_{\psi}\left(\frac{\partial \phi}{\partial \psi}\right)^{2}-\partial_{\psi}^{2}\left(\frac{\partial \phi}{\partial \alpha} \frac{\partial \phi}{\partial \psi}\right)\right) \\
& +\rho_{s}^{2}\left(\partial_{\alpha}^{2}\left(\frac{\partial \phi}{\partial \alpha} \frac{\partial \phi}{\partial \psi}\right)-\partial_{\alpha} \partial_{\psi}\left(\frac{\partial \phi}{\partial \alpha}\right)^{2}\right) .
\end{aligned}
$$

Using the relation $\phi=\langle\phi\rangle_{\alpha}+\delta \phi$, the integration over $\alpha$ of (A7) leads to 


$$
C_{i}\langle[\delta \phi, \bar{\triangle} \delta \phi]\rangle_{\alpha}=-C_{i} \delta_{b}^{2} \partial_{\psi}^{2}\left\langle\frac{\partial \delta \phi}{\partial \alpha} \frac{\partial \delta \phi}{\partial \psi}\right\rangle_{\alpha} .
$$

(ii) The last term in (A6) is connected to the Poisson bracket $[\phi, P]=\partial_{\alpha}\left(P \frac{\partial \phi}{\partial \psi}\right)-\partial_{\psi}\left(P \frac{\partial \phi}{\partial \alpha}\right)$. Taking into account the expression of $\phi$ and $\mathrm{P}$ in the form of the sum of a mean value plus a fluctuation, we then have

$$
\left\langle\frac{3}{2} \bar{\triangle}[\delta \phi, \delta P]\right\rangle_{\alpha}=\frac{\delta_{b}^{2}}{4} \partial_{\psi}^{2}\left(\frac{\partial \delta Q}{\partial \psi}\right),
$$

where $\delta Q$ is the heat flux.

(iii) Finally, we introduce the quantity $J=[\phi, \bar{\triangle} P]$ $-[\bar{\triangle} \phi, P]$. Substituting $\bar{\triangle}=\rho_{s}^{2} \partial_{\alpha}^{2}$ in the previous equation leads to

$$
\frac{J}{\rho_{s}^{2}}=\partial_{\alpha}^{2}\left(\frac{\partial P}{\partial \alpha} \frac{\partial \phi}{\partial \psi}+\frac{\partial P}{\partial \psi} \frac{\partial \phi}{\partial \alpha}\right)-2 \partial_{\alpha} \partial_{\psi}\left(\frac{\partial \phi}{\partial \alpha} \frac{\partial P}{\partial \alpha}\right) .
$$

Substituting now $\bar{\triangle}=\delta_{b}^{2} \partial_{\psi}^{2}$ in $J$ reads as

$$
\frac{J}{\delta_{b}^{2}}=\partial_{\psi}^{2}\left(\frac{\partial P}{\partial \alpha} \frac{\partial \phi}{\partial \psi}+\frac{\partial P}{\partial \psi} \frac{\partial \phi}{\partial \alpha}\right)-2 \partial_{\alpha} \partial_{\psi}\left(\frac{\partial \phi}{\partial \psi} \frac{\partial P}{\partial \psi}\right) .
$$

Finally, by separating mean values and fluctuations (A10) and (A11) give rise to the following expression:

$$
\langle[\delta \phi, \bar{\triangle} \delta P]-[\bar{\triangle} \delta \phi, \delta P]\rangle_{\alpha}=\delta_{b}^{2} \partial_{\psi}^{2}\left\langle\frac{\partial \delta P}{\partial \alpha} \frac{\partial \delta \phi}{\partial \psi}+\frac{\partial \delta P}{\partial \psi} \frac{\partial \delta \phi}{\partial \alpha}\right\rangle_{\alpha}
$$

Replacing Eqs. (A8), (A9), and (A12) in (A5) leads to the expected formula. After a double integration over the $\psi$ variable, we obtain

$$
\begin{aligned}
\frac{\partial\langle\phi\rangle_{\alpha}}{\partial t}= & \left\langle\frac{\partial \delta \phi}{\partial \alpha} \frac{\partial \delta \phi}{\partial \psi}\right\rangle_{\alpha}-\frac{3}{8 C_{i}}\left\langle\frac{\partial \delta P}{\partial \alpha} \frac{\partial \delta \phi}{\partial \psi}+\frac{\partial \delta P}{\partial \psi} \frac{\partial \delta \phi}{\partial \alpha}\right\rangle_{\alpha} \\
& +\frac{1}{4 C_{i}} \partial_{\psi}\langle\delta Q\rangle_{\alpha} .
\end{aligned}
$$

${ }^{1}$ K. H. Burrell, T. N. Carlstrom, E. J. Doyle, D. Finkenthal, P. Gohil, R. J. Groebner, D. L. Hillis, J. Kim, H. Matsumoto, R. A. Moyer, T. H. Osborne, C. L. Rettig, W. A. Peebles, T. L. Rhodes, H. StJohn, R. D. Stambaugh, M. R. Wade, J. G. Watkins, and DIII-D Team, Plasma Phys. Controlled Fusion 34, 1859 (1992).
${ }^{2}$ G. S. Xu, B. N. Wan, H. Q. Wang, H. Y. Guo, H. L. Zhao, A. D. Liu, V. Naulin, P. H. Diamond, G. R. Tynan, M. Xu, R. Chen, M. Jiang, P. Liu, N. Yan, W. Zhang, L. Wang, S. C. Liu, and S. Y. Ding, Phys. Rev. Lett. 107, 125001 (2011).

${ }^{3}$ G. D. Conway, C. Angioni, F. Ryter, P. Sauter, J. Vicente, and ASDEX Upgrade Team, Phys. Rev. Lett. 106, 065001 (2011).

${ }^{4}$ M. N. Rosenbluth and F. L. Hinton, Phys. Rev. Lett. 80, 724 (1998).

${ }^{5}$ D. K. Gupta, R. J. Fonck, G. R. McKee, D. J. Schlossberg, and M. W. Shafer, Phys. Rev. Lett. 97, 125002 (2006).

${ }^{6}$ Y. Hamada, T. Watari, A. Nishizawa, K. Narihara, Y. Kawasumi, T. Ido, M. Kojima, K. Toi, and JIPPT-IIU Group, Phys. Rev. Lett. 96, 115003 (2006).

${ }^{7}$ P. A. Polizer, Phys. Rev. Lett. 84, 1192 (2000).

${ }^{8}$ H. Bigliari, P. H. Diamond, and P. W. Terry, Phys. Fluids B 2, 1 (1990).

${ }^{9}$ A. Hasegawa and K. Mima, Phys. Rev. Lett. 39, 205 (1977).

${ }^{10}$ J. G. Charney, Geophys. Publ. Oslo 17, 1 (1948).

${ }^{11}$ W. Dorland and G. W. Hammett, Phys. Fluids B 5, 812 (1993).

${ }^{12}$ A. Hasegawa and M. Wakatani, Phys. Rev. Lett. 50, 682 (1983).

${ }^{13}$ R. Numata, R. Ball, and R. L. Dewar, Phys. Plasmas 14, 102312 (2007).

${ }^{14}$ A. V. Pushkarev, W. J. T. Bos, and S. V. Nazarenko, Phys. Plasmas 20, 042304 (2013).

${ }^{15}$ I. R. Goumiri, C. W. Rowley, Z. Ma, D. A. A. Gates, J. A. Krommes, and J. B. Parker, Phys. Plasmas 20, 042501 (2013).

${ }^{16}$ K. Itoh, S. I. Itoh, P. H. Diamond, T. S. Hahm, A. Fujisawa, G. R. Tynan, M. Yagi, and Y. Nayashima, Phys. Plasmas 13, 055502 (2006).

${ }^{17}$ P. H. Diamond, A. Hasegawa, and K. Mima, Plasma Phys. Controlled Fusion 53, 124001 (2011).

${ }^{18}$ J. G. Charney and P. G. Drazin, J. Geophys. Res. 66, 83, doi:10.1029/ JZ066i001p00083 (1961).

${ }^{19}$ P. H. Diamond, O. D. Gurcan, T. S. Hahm, K. Miki, Y. Kosuga, and X. Garbet, Plasma Phys. Controlled Fusion 50, 124018 (2008).

${ }^{20}$ B. N. Rogers, W. Dorland, and M. Kotschenreuther, Phys. Rev. Lett. 85, 5336 (2000).

${ }^{21}$ E. J. Kim and P. H. Diamond, Phys. Plasmas 9, 4530 (2002).

${ }^{22}$ B. B. Kadomtsev and O. P. Pogutse, Nucl. Fusion 11, 67 (1971).

${ }^{23}$ A. Ghizzo, M. El Mounden, D. Del Sarto, X. Garbet, and Y. Sarazin, Transp. Theor. Stat. Phys. 40, 382 (2011).

${ }^{24}$ A. Ghizzo, D. Del Sarto, X. Garbet, and Y. Sarazin, Phys. Plasmas 17, 092501 (2010).

${ }^{25}$ L. Wang and T. S. Hahm, Phys. Plasmas 16, 062309 (2009).

${ }^{26}$ M. Artun, W. M. Tang, and G. Rewoldt, Phys. Plasmas 2, 3384 (1995).

${ }^{27}$ D. Zarsozo, X. Garbet, Y. Sarazin, R. Dumont, and V. Grandgirard, Phys. Plasmas 19, 022102 (2012).

${ }^{28}$ W. M. Tang, J. C. Adam, and D. W. Ross, Phys. Fluids 20, 430 (1977).

${ }^{29}$ E. Sonnendrücker, J. Roche, P. Bertrand, and A. Ghizzo, J. Comput. Phys. 149, 201 (1999).

${ }^{30}$ A. Ghizzo, P. Bertrand, M. Shoucri, E. Fijalkow, and M. R. Feix, Phys. Fluids B 5, 4312 (1993).

${ }^{31}$ G. Manfredi, M. Shoucri, R. O. Dendy, A. Ghizzo, and P. Bertrand, Phys. Plasmas 3, 202 (1996).

${ }^{32}$ N. Winsor, J. L. Johnson, and J. M. Dawson, Phys. Fluids 11, 2448 (1968).

${ }^{33}$ K. Hallatschek and D. Biskamp, Phys. Rev. Lett. 86, 1223 (2001).

${ }^{34}$ B. Scott, Phys. Lett. A 320, 53 (2003).

${ }^{35}$ K. Miki, Y. Kishimoto, J. Li, and N. Migato, Phys. Plasmas 15, 052309 (2008).

${ }^{36}$ Z. Gao, Phys. Plasmas 20, 032501 (2013).

${ }^{37}$ P. H. Diamond and Y. B. Kim, Phys. Fluids B 3, 1626 (1991).

${ }^{38}$ A. Ghizzo and F. Palermo, Phys. Plasmas 22, 082304 (2015). 